\title{
A Labdane Diterpene from the Aerial Parts of Egletes viscosa Less
}

\author{
Francisco Artur e Silva-Filho, ${ }^{a}$ Mary Anne S. Lima, ${ }^{* a}$ Antonio Marcos E. Bezerra, ${ }^{b}$ \\ Raimundo Braz Filho ${ }^{c}$ and Edilberto R. Silveira ${ }^{a}$ \\ ${ }^{a}$ Curso de Pós-Graduação em Química Orgânica, Universidade Federal do Ceará, CP 12.200 \\ 60.021-940, Fortaleza-CE, Brazil \\ ${ }^{b}$ Departamento de Fitotecnia, Universidade Federal do Ceará, CP 12168 \\ 60356-760 Fortaleza-CE, Brazil \\ ${ }^{c}$ Universidade Estadual do Norte Fluminense, Setor de Química de Produtos Naturais, \\ 28013-600 Campos-RJ, Brazil
}

Um novo labdano glicosilado, 8 $\alpha$-hidroxilabd-14(15)-eno-13(S)-O- $\beta$-D-ribopiranosídeo, foi isolado das partes aéreas de Egletes viscosa Less, juntamente com o 13-epi-esclareol, barbatol, tarapacol, espinasterol, ternatina e triacontano. As estruturas dos compostos isolados foram elucidadas pelo uso de métodos espectrométricos e preparação de derivados químicos.

A new labdane glycoside, $8 \alpha$-hydroxylabd-14(15)-ene-13(S)-O- $\beta$-D-ribopyranoside, was isolated from the aerial parts of Egletes viscosa Less, along with 13-epi-sclareol, barbatol, tarapacol, spinasterol, ternatin and triacontane. The structures of the isolated compounds were elucidated by the use of spectrometric methods and preparation of chemical derivatives.

Keywords: Asteraceae, Egletes viscosa, diterpene glycoside, 8$\alpha$-hydroxylabd-14(15)-ene13(S)-O- $\beta$-D-ribopyranoside

\section{Introduction}

Egletes viscosa Less (Asteraceae) is an annual herb mostly native to intertropical Americas. It is known in the Northeast of Brazil as "macela" or "macela-da-terra" and grows throughout the margins of river and lakes as soon as the raining season ends. The flower buds are largely sold in the herbal stores and used in the folk medicine as emmenagogue, diaphoretic, ${ }^{1}$ stomachic and antidiarrhoeal. $^{2}$

Previous studies with E. viscosa have reported the isolation of biologically active flavonoids and diterpenoids. ${ }^{3-14}$ Recently, Lee et al reported the isolation of labdane diterpene glycosides and flavonoids from the entire plant native from Peru. ${ }^{15}$

In continuation of the work with this species, we report now the isolation of the new labdane glycoside $8 \alpha$ hydroxylabd-14(15)-ene-13(S)-O- $\beta$-D-ribopyranoside 1 in addition to 13 -epi-sclareol, ${ }^{16}$ barbatol, ${ }^{17}$ tarapacol, ${ }^{18}$ spinasterol, ${ }^{19}$ ternatin, ${ }^{7,20-23}$ and triacontane from the aerial

*e-mail: mary@dqoi.ufc.br parts of E. viscosa. The complete proton and carbon assignments of the known compounds and of the new diterpene 1, including the unpublished ${ }^{13} \mathrm{C}$ and ${ }^{1} \mathrm{H}$ NMR data of the new derivatives $\mathbf{2 - 4}$ were accomplished by the use of a series of 2D NMR experiments such as ${ }^{1} \mathrm{H}-{ }^{1} \mathrm{H}$ COSY, HMQC and HMBC.

\section{Results and Discussion}

Compound $\mathbf{1}$ was isolated as a white solid. Its molecular formula was determined as $\mathrm{C}_{25} \mathrm{H}_{44} \mathrm{O}_{6}$ from the NMR and EImass spectroscopy measurements $(\mathrm{m} / \mathrm{z}=440)$. The absorption band in the IR spectrum at $3400 \mathrm{~cm}^{-1}$ was consistent with hydroxyl groups. Inspection of the ${ }^{1} \mathrm{H}$ NMR spectrum indicated the presence of three angular methyl groups at $\delta$ 0.81 (s, H-19 and H-20) and 0.88 (s, H-18), two methyls attached to oxygenated tertiary carbons at $\delta 1.11(\mathrm{~s}, \mathrm{H}-17)$ and 1.35 (s, H-16), and an anomeric hydrogen at $\delta 4.32\left(\mathrm{~d}, J 6.4 \mathrm{~Hz}, \mathrm{H}-1^{\prime}\right)$. Moreover, the presence of a vinyl moiety was suggested by the signals at $\delta 5.92(\mathrm{dd}, J$ 17.8, 10.9, H-14); 5.22 (dd, $J 17.8,1.2$, H-15a) and 5.17 (dd, $J 10.9,1.2, \mathrm{H}-15 \mathrm{~b})$. The ${ }^{13} \mathrm{C}$ NMR spectral data of 1 confirmed 
the $\beta$-ribopyranosyl unit by the signals at $\delta 98.6\left(\mathrm{C}-1^{\prime}\right), 73.1$ $\left(\mathrm{C}-3^{\prime}\right), 71.3\left(\mathrm{C}-2^{\prime}\right), 68.2\left(\mathrm{C}-4^{\prime}\right)$ and $65.1\left(\mathrm{C}-5^{\prime}\right) ;^{24}$ together with 20 carbon signals ascribable to the aglycone, that were assigned to five methyls, nine methylenes, seven methine and four non-hydrogenated, two of which oxygen bearing carbons (C-13 and C-8).

The unequivocal assignment of NMR spectral data of compound $\mathbf{1}$ shown in Table $\mathbf{1}$ was established by data analysis from COSY, HMQC and HMBC experiments. The spectral data attributed to the aglycone unit showed a very similar feature to those described for sclareol..$^{25}$ The HMBC experiment confirmed the position of glycosylation at $\mathrm{C}-13$ by the correlations of the anomeric hydrogen at $\delta 4.32\left(\mathrm{H}-1^{\prime}\right)$ and both methylidene hydrogens at $\delta 5.22$ and 5.17 (H-15a and H-15b) with the oxygenated carbon at $\delta 80.6$ (C-13). The decaline ring system was characterized by long-range correlations of both methyl groups at $\delta 0.88(\mathrm{H}-18)$ and $0.81(\mathrm{H}-19)$ with the carbons at $\delta 42.0(\mathrm{C}-3)$ and $56.3(\mathrm{C}-5)$, as well as the methyl groups at $\delta 0.81(\mathrm{H}-20)$ with the carbons at $\delta 39.9(\mathrm{C}-1), 56.3(\mathrm{C}-5)$ and 61.7 (C-9). Further, pertinent correlations of the methyl group at $d 1.11(\mathrm{H}-$ 17) with the carbons at $\delta 43.7$ (C-7), 61.7 (C-9) and 74.0 (C-8) allowed to confirm the allocation of the hydroxyl group at C-8.

A survey in the literature revealed that either $(+)$ or (-) optical rotations can be observed for any kind of sclareol glycosides either from the normal or the enantiomer series, e.g., both the (13R)-labd-14(15)-ene8,13-diol-13-O- $\beta$-fucopyranoside or its aglycone belonging to the normal series show minus signal for their optical rotations, ${ }^{26}$ but the ent-sclareol [ent-(13R)labd-14(15)-ene-8,13-diol] showed $(+)$ signal while its $13-O-\beta$-xylopyranoside derivative showed (-) optical rotation. ${ }^{27}$ The literature also reports the ${ }^{13} \mathrm{C}$ NMR data for sclareol, ${ }^{25}$ ent-sclareol ${ }^{27}$ and 13-epi-sclareol ${ }^{16}$. The latter, obtained from Egletes viscosa of Peruvian origin, showed $\left.[\alpha]_{\mathrm{D}}^{20}+4.5^{\circ}\left(c 0.11, \mathrm{CHCl}_{3}\right)\right)^{15}$

In this work a sclareol entity, 5, was isolated. Comparison of its optical rotation, $+5.0^{\circ}\left(c 0.36, \mathrm{CHCl}_{3}\right)$, as well as comparison of the ${ }^{13} \mathrm{C}$ NMR data with those reported in the literature allowed its identification as 13-

Table 1. NMR assignments for the compounds 1-5 (500/125 MHz)

\begin{tabular}{|c|c|c|c|c|c|c|c|c|c|}
\hline \multirow[t]{2}{*}{ Carbon } & \multicolumn{4}{|c|}{${ }^{1} \mathrm{H}$ NMR } & \multicolumn{5}{|c|}{${ }^{13} \mathrm{C}$ NMR } \\
\hline & 1 & 2 & 3 & 4 & 1 & 2 & 3 & 4 & 5 \\
\hline 1 & 1.681 .12 & 1.690 .96 & 0.901 .56 & 1.570 .88 & 39.9 & 41.3 & 39.8 & 39.7 & 39.9 \\
\hline 2 & 1.621 .40 & 1.661 .29 & 1.58 & 1.621 .29 & 20.2 & 21.6 & 20.5 & 20.3 & 20.7 \\
\hline 3 & 1.401 .18 & 1.401 .18 & 1.131 .30 & 1.391 .13 & 42.0 & 43.3 & 42.4 & 42.2 & 42.2 \\
\hline 4 & & & & & 39.3 & 40.5 & 39.2 & 39.1 & 32.7 \\
\hline 5 & $0.94(\mathrm{dd}, J 11.9,2.1 \mathrm{~Hz})$ & $0.96(\mathrm{dd}, J 11.9,2.1 \mathrm{~Hz})$ & $0.92(\mathrm{~m})$ & $0.90(\mathrm{~m})$ & 56.3 & 57.7 & 56.3 & 56.3 & 56.2 \\
\hline 6 & 1.581 .30 & 1.541 .27 & 1.211 .39 & $1.58 \quad 1.41$ & 19.3 & 20.3 & 19.1 & 18.6 & 19.3 \\
\hline 7 & 1.821 .40 & 1.711 .54 & 1.341 .80 & $1.79 \quad 1.42$ & 43.7 & 43.4 & 43.3 & 42.5 & 44.9 \\
\hline 8 & & & & & 74.0 & 75.4 & 74.4 & 74.4 & 74.4 \\
\hline 9 & 1.30 & 1.05 & 1.00 & 1.03 & 61.7 & 63.3 & 62.0 & 61.9 & 62.0 \\
\hline 10 & & & & & 32.9 & 34.3 & 33.4 & 33.4 & 39.4 \\
\hline 11 & 1.651 .45 & 1.631 .44 & 1.381 .56 & $1.47 \quad 1.27$ & 18.2 & 19.6 & 18.6 & 18.5 & 18.6 \\
\hline 12 & 1.821 .62 & 1.811 .44 & 1.551 .75 & $1.47 \quad 1.73$ & 44.8 & 44.8 & 45.2 & 43.1 & 44.3 \\
\hline 13 & & & & & 80.6 & 81.9 & 80.9 & 81.0 & 75.2 \\
\hline 14 & $5.92(\mathrm{dd}, J 17.8,10.9 \mathrm{~Hz})$ & $1.59(\mathrm{dd}, J 7.5,2.0 \mathrm{~Hz})$ & $5.69(\mathrm{dd}, J 17.2,10.6 \mathrm{~Hz})$ & 1.591 .38 & 143.2 & 32.2 & 142.1 & 31.4 & 145.4 \\
\hline 15 & $\begin{array}{l}5.22(\mathrm{dd}, J 17.8,1.2 \mathrm{~Hz}) \\
5.17(\mathrm{dd}, J 10.9,1.2 \mathrm{~Hz})\end{array}$ & $0.92(\mathrm{t}, J 7.4 \mathrm{~Hz})$ & $\begin{array}{l}5.20(\mathrm{dd}, J 17.8,1.3 \mathrm{~Hz}) \\
5.17(\mathrm{dd}, J 10.6,1.3 \mathrm{~Hz})\end{array}$ & $0.82(\mathrm{t}, J 7.0 \mathrm{~Hz})$ & 114.1 & 8.9 & 115.3 & 8.2 & 112.0 \\
\hline 16 & $1.35(\mathrm{~s})$ & $1.20(\mathrm{~s})$ & $1.30(\mathrm{~s})$ & $1.13(\mathrm{~s})$ & 22.2 & 24.2 & 24.4 & 23.4 & 24.7 \\
\hline 17 & $1.11(\mathrm{~s})$ & $1.14(\mathrm{~s})$ & $1.14(\mathrm{~s})$ & $1.16(\mathrm{~s})$ & 22.7 & 24.3 & 24.5 & 24.8 & 24.3 \\
\hline 18 & $0.88(\mathrm{~s})$ & $0.88(\mathrm{~s})$ & $0.85(\mathrm{~s})$ & $0.85(\mathrm{~s})$ & 32.7 & 34.0 & 33.5 & 33.5 & 33.6 \\
\hline 19 & $0.81(\mathrm{~s})$ & $0.81(\mathrm{~s})$ & $0.77(\mathrm{~s})$ & $0.76(\mathrm{~s})$ & 20.7 & 22.0 & 21.6 & 21.6 & 21.7 \\
\hline 20 & $0.81(\mathrm{~s})$ & $0.83(\mathrm{~s})$ & $0.75(\mathrm{~s})$ & $0.77(\mathrm{~s})$ & 14.8 & 16.3 & 15.7 & 15.7 & 15.4 \\
\hline $1^{\prime}$ & $4.32(\mathrm{~d}, J 6.4 \mathrm{~Hz})$ & $4.42(\mathrm{~d}, J 6.4 \mathrm{~Hz})$ & $4.56(\mathrm{~d}, J 7.3 \mathrm{~Hz})$ & $4.62(\mathrm{~d}, J 6.5 \mathrm{~Hz})$ & 98.6 & 98.9 & 96.5 & 95.9 & \\
\hline $2^{\prime}$ & 3.53 & 3.52 & 5.22 & 5.20 & 71.3 & 74.5 & 69.6 & 69.8 & \\
\hline $3^{\prime}$ & 3.51 & 3.51 & 5.03 & 5.03 & 73.1 & 72.8 & 70.6 & 70.7 & \\
\hline $4^{\prime}$ & 3.78 & 3.79 & 4.56 & 5.24 & 68.2 & 69.6 & 67.9 & 67.8 & \\
\hline $5^{\prime}$ & $\begin{array}{l}3.79 \\
3.49\end{array}$ & $\begin{array}{c}3.98(\mathrm{dd}, J 13.2,2.5 \mathrm{~Hz}) \\
3.59(\mathrm{~d}, J 13.2 \mathrm{~Hz})\end{array}$ & $\begin{array}{c}3.60(\mathrm{~d}, J 12.5 \mathrm{~Hz}) \\
3.95(\mathrm{dd}, J 12.5,2.5 \mathrm{~Hz})\end{array}$ & & 65.1 & 66.5 & 63.7 & 63.7 & \\
\hline $1^{\prime \prime}$ & & & & & & & 169.5 & 169.5 & \\
\hline $2^{\prime \prime}$ & & & $2.13(\mathrm{~s})$ & & & & 21.0 & 21.0 & \\
\hline $3^{\prime \prime}$ & & & & & & & 170.4 & 170.4 & \\
\hline $4^{\prime \prime}$ & & & $2.01(\mathrm{~s})$ & & & & 20.8 & 20.8 & \\
\hline $5^{\prime \prime}$ & & & & & & & 170.6 & 170.5 & \\
\hline $6^{\prime \prime}$ & & & $2.05(\mathrm{~s})$ & & & & 21.1 & 21.1 & \\
\hline
\end{tabular}


epi-sclareol, based mainly on the $\mathrm{C}-13$ chemical shift $(\delta$ 75.2 ), which in the case of sclareol (or ent-sclareol) is found at $\delta$ 73.0. (Table 1). ${ }^{16,25,27}$ Thus, according to the latter evidences we suggest that the diterpenes here reported belong to the 13-epi-sclareol series whose stereochemistry is depicted in Figure 1.

On the basis of the above spectroscopic data, in addition to all optical rotation considerations, compound 1 was identified as the novel labdane diterpenoid $8 \alpha$ hydroxylabd-14(15)-ene-13(S)-O- $\beta$-D-ribopyranoside. Chemical derivatization by usual methods was used to confirm the proposed structure and yielded the new labdane derivatives $\mathbf{2 - 4}$, to which the ${ }^{1} \mathrm{H}$ and ${ }^{13} \mathrm{C}$ NMR data are presented in Table $\mathbf{1}$.

The isolated diterpenes $8 \alpha$-hydroxylabdan-14(15)-en13(S)-O- $\beta$-D-ribopyranoside 1, barbatol, tarapacol, as well as the derivatives $\mathbf{2 - 4}$, were tested for their cytotoxicity using five tumor cell lines: two human leukemias (HL-60 and CEM), human breast adenocarcinoma (MCF-7), human colon adenocarcinoma (HCT-8) and murine melanoma (B16), but none showed any cytotoxic activity.

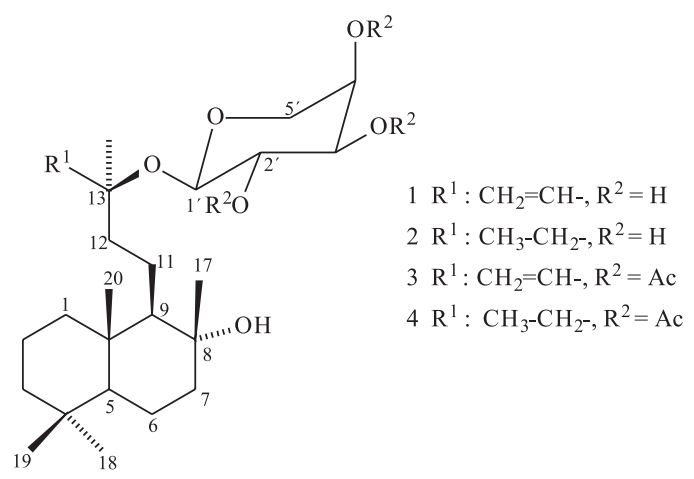

Figure 1. Structures of $8 \alpha$-hydroxylabd-14(15)-ene-13(S)-O- $\beta$-Dribopyranoside (1), $8 \alpha$-hydroxylabd-13(S)-O- $\beta$-D-ribopyranoside (2), $8 \alpha$-hydroxylabd-14(15)-ene-13(S)-O- $\beta$-D-2', $3^{\prime}, 4^{\prime}$-triacetylribopyranoside (3) and $8 \alpha$-hydroxylabd-13(S)-O- $\beta-D-2^{\prime}, 3^{\prime}, 4^{\prime}$ - triacetylribopyranoside (4).

\section{Experimental}

\section{General procedures}

Melting points were obtained on a Mettler FP82HT apparatus and are uncorrected. IR spectra were recorded using a Perkin Elmer 1000 FT-IR spectrophotometer. Optical rotations were measured on a Perkin Elmer 341 polarimeter. The mass spectra were obtained on a Hewlett-Packard 5971 mass spectrometer by electron impact ionization $(70 \mathrm{eV}) .{ }^{1} \mathrm{H}$ and ${ }^{13} \mathrm{C}$ NMR spectra were recorded on a Bruker Avance DRX-500 $\left(500 \mathrm{MHz}\right.$ for ${ }^{1} \mathrm{H}$ and $125 \mathrm{MHz}$ for ${ }^{13} \mathrm{C}$ ); chemical shifts are given in ppm relative to residual $\mathrm{CHCl}_{3}$ (7.24 and $77.0 \mathrm{ppm}$ ). Silica Gel 60 (Merck, 230-400 mesh) was used for analytical
TLC. Silica gel 60 (Merck, $60 \mathrm{~F}_{254}, 0.2 \mathrm{~mm}$ ) was used for column chromatography. All compounds were visualized on TLC by spraying with vanillin/perchloric $\mathrm{acid} / \mathrm{EtOH}$ followed by heating.

\section{Plant material}

Aerial parts of cultivated Egletes viscosa Less were harvested from garden beds at the Departamento de Fitotecnia of the Universidade Federal do Ceará, Fortaleza, Ceará State. Voucher specimens (36694) have been identified by Dr. Edson Paula Nunes and deposited at the Herbário Prisco Bezerra (EAC), Departamento de Biologia, Universidade Federal do Ceará, Fortaleza, CE, Brazil.

\section{Extraction and isolation}

Dried aerial parts of $E$. viscosa $(1.35 \mathrm{~kg})$ were pulverized and extracted with hexane at room temperature $(3 \times 8 \mathrm{~L})$. The solvent was removed under reduced pressure to give the correspondent extract and the mark obtained after hexane extraction was reextracted with EtOH $(3 \times 8 \mathrm{~L})$.

The hexane extract $(12.7 \mathrm{~g})$ was coarsely fractionated on a Silica gel column by elution with hexane, $\mathrm{CHCl}_{3}, \mathrm{EtOAc}$ and $\mathrm{MeOH}$. Chromatography of the hexane fraction $(1.87 \mathrm{~g})$ using hexane/EtOAc mixtures with increasing polarity yielded triacontane (38.7 mg, $0.3 \%$ ). Successive flash chromatography of the $\mathrm{CHCl}_{3}$ fraction $(3.0 \mathrm{~g}$ ) using $0-100 \%$ hexane/EtOAc mixtures yielded spinasterol (120.0 mg, 0.94 $\%)$ and 13-epi-sclareol 5 (32.0 mg, (0.25\%). The EtOAc fraction $(2.0 \mathrm{~g})$ was further purified over Sephadex LH-20 by elution with $\mathrm{MeOH}$ to yield tarapacol $(8.9 \mathrm{mg}, 0.07 \%)$.

The EtOH extract $(15.0 \mathrm{~g})$ was redissolved in a mixture of $\mathrm{MeOH}: \mathrm{H}_{2} \mathrm{O}(1: 1 \mathrm{v} / \mathrm{v})$ and submitted to liquidliquid partition with petrol ether, $\mathrm{CHCl}_{3}, \mathrm{EtOAc}$ and $\mathrm{MeOH}$ to give four fractions. Chromatography on a Silica gel column of the petrol ether fraction $(2.4 \mathrm{~g})$ by elution with hexane, $\mathrm{CHCl}_{3}$, EtOAc and $\mathrm{MeOH}$ yielded the correspondent sub-fractions. Flash chromatography of the $\mathrm{CHCl}_{3}$ sub-fraction $(590.5 \mathrm{mg})$ using hexane/ EtOAc mixtures with increasing polarity, afforded 80 fractions. Preparative chromatography of the fraction $68-73(43.5 \mathrm{mg})$ by elution with a mixture of $\mathrm{CHCl}_{3} /$ EtOAc 1:1 yielded compound $1(19.0 \mathrm{mg})$. TLC preparative chromatography by elution with hexane/ EtOAc $(6: 4 \mathrm{v} / \mathrm{v})$ of fraction 30-36 (36.0 mg, $0.24 \%)$ yielded barbatol $(8.2 \mathrm{mg}, 0.05 \%)$. The EtOAc subfraction $(3.0 \mathrm{~g})$ was further purified over Sephadex LH20 by elution with $\mathrm{MeOH}$ to afford ternatin $(6.1 \mathrm{mg}$, $0.04 \%)$. 


\section{Hydrogenation of 1}

Compound $1(12.0 \mathrm{mg})$ dissolved in EtOAc $(5.0 \mathrm{~mL})$ was added to a suspension containing $\mathrm{Pd} / \mathrm{Rh}(2.0 \mathrm{mg})$ in $\mathrm{MeOH}(10.0 \mathrm{~mL})$, that was previously saturated with $\mathrm{H}_{2}$. The mixture was stirred at room temperature during 30 minutes. Usual work up and $\mathrm{SiO}_{2}$ column chromatography using hexane $/ \mathrm{CHCl}_{3} 2: 1$ yielded compound 2 (10.0 mg, $83.3 \%$ ) as a white solid.

\section{Acetylation of $\mathbf{1}$}

To a solution of compound $\mathbf{1}(5.0 \mathrm{mg})$ in pyridine $(1.0 \mathrm{~mL})$ were added $\mathrm{Ac}_{2} \mathrm{O}(1.0 \mathrm{~mL})$ and a catalytic amount of DMAP. The mixture was stirred for 30 minutes at room temperature. Subsequent workup afforded a residue that was chromatographed using hexane/ $\mathrm{CHCl}_{3}$ (3:1) as eluent to yield compound 3 (6.0 $\mathrm{mg}, 94.0 \%)$ as a yellowish solid.

\section{Hydrogenation of $\mathbf{2}$}

Compound $2(10.0 \mathrm{mg})$ dissolved in EtOAc $(4.0 \mathrm{~mL})$ was added to a suspension containing $\mathrm{Pd} / \mathrm{Rh}(2.0 \mathrm{mg})$ in $\mathrm{MeOH}(80.0 \mathrm{~mL})$ that was previously saturated with $\mathrm{H}_{2}$. The mixture was stirred at room temperature during 30 minutes. Usual work up and $\mathrm{SiO}_{2}$ column chromatography using hexane $/ \mathrm{CHCl}_{3} 2: 1$ yielded compound $4(8.0 \mathrm{mg}$, $80.0 \%$ ) as a white solid.

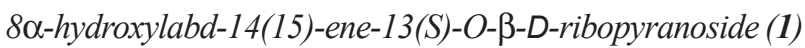

White solid; m.p. $89.7-91.0^{\circ} \mathrm{C} ;[\alpha]_{\mathrm{D}}^{20}:+10.0^{\circ}(\mathrm{MeOH} ; c$ 0.02); IR (film) $v_{\max } \mathrm{cm}^{-1}: 3400,2927,2867,1647,1459,1388$, 1131, 1073, 1002, 918, 784; EIMS $m / z$ (rel. int.): $440\left[\mathrm{M}^{+}\right](3)$, 290 (8), 273 (15), 257 (6), 245 (3), 232 (2), 217 (8), 203 (13), 191 (59), 177 (75), 163 (25), 149 (14), 137 (14), 123 (20), 109 (39), 95 (58), 81 (79), 69 (44), 43 (100), 41 (71).

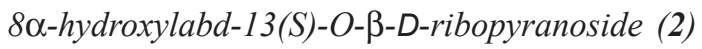

White solid; m.p. $71.7-74.2^{\circ} \mathrm{C} ;[\alpha]_{\mathrm{D}}^{20}:+7.0^{\circ}(\mathrm{MeOH}$; $c 0.28$ ); IR (film) $v_{\max } \mathrm{cm}^{-1}: 3400,2927,2867,1647,1459$, 1388, 1131, 1073, 1002, 918, 784; EIMS m/z (rel. int.): $442\left[\mathrm{M}^{+}\right](4), 292$ (27), 275 (100), 259 (17), 245 (41), 234 (17), 221 (14), 205 (21), 191 (43), 177 (57), 163 (25), 149 (30), 137 (36), 123 (38), 109 (78), 95 (68), 95 (69), 69 (60), 43 (74), 41 (37).

$8 \alpha$-hydroxylabd-14(15)-ene-13(S)-O- $\beta-D-2^{\prime}, 3^{\prime}, 4^{\prime}$ triacetylribopyranoside (3)

Colorless resin; $[\alpha]_{\mathrm{D}}{ }^{20}:+15.0^{\circ}\left(\mathrm{CHCl}_{3} ; c\right.$ 0.02); IR (film) $v_{\max } / \mathrm{cm}^{-1}: 3366,2925,2852,1748,1493,1380$, 1347, 1131, 1092, 1073, 1002, 944, 823, 702; EIMS $\mathrm{m} / \mathrm{z}$ (rel. int.): $566\left[\mathrm{M}^{+}\right](5), 292(14), 275$ (63), 259 (11), 245 (31), 234 (3), 221 (7), 204 (27), 191 (37),
177 (56), 163 (27), 149 (31), 137 (32), 123 (28), 109 (42), 95 (58), 73 (83), 69 (71), 43 (100), 41 (52).

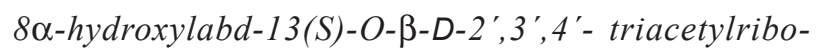
pyranoside (4)

Colorless resin; $[\alpha]_{\mathrm{D}}^{20}:+13.0^{\circ}\left(\mathrm{CHCl}_{3} ; c 0.02\right)$; IR (film) $v_{\max } / \mathrm{cm}^{-1}: 3322,2930,2823,1752,1333,1147,1096,1081$, 952, 735; EIMS m/z (rel. int.): 568 [M'](3), 292 (25), 275 (79), 259 (100), 245 (25), 234 (4), 217 (15), 199 (25), 191 (31), 177 (38), 157 (39), 151 (13), 139 (82), 123 (24), 109 (51), 97 (63), 81 (31), 69 (32), 43 (100), 41 (7).

\section{Determination of Cytotoxicity}

All compounds ( 0.39 to $25 \mu \mathrm{g} / \mathrm{mL}$ ) were tested for cytotoxic activity at the Departamento de Fisiologia e Farmacologia of the Universidade Federal do Ceará. Five tumor cell lines were used (National Cancer Institute, Bethesda, MD, USA): B=16 (murine melanoma), HCT-8 (human colon), MCF-7 (human breast), CEM and HL-60 (leukemia) after 72 hours of incubation. Doxorubicin (0.01 to $0.58 \mu \mathrm{g} / \mathrm{mL}$ ) was used as a positive control. The general viability of cultured cells was determined by reduction of the yellow dye 3-(4,5-dimethyl-2-thiazolyl)-2,5-diphenyl$2 \mathrm{H}$-tetrazolium bromide (MTT) to a blue formazan product as described by Mosmann. ${ }^{28}$

\section{Acknowledgments}

The authors are grateful to CNPq/CAPES/PRONEX/ FUNCAP/FINEP for the fellowships and financial support.

\section{Supplementary Information}

Supplementary data including spectral data of all compounds are available free of charge at http:// jbcs.sbq.org.br, as PDF file.

\section{References}

1. Braga, R.; Plantas do Nordeste, especialmente do Ceará, Imprensa Oficial: Fortaleza, Brazil, 1960.

2. Correia, P.; Dicionário de Plantas Úteis do Brasil e das Exóticas Cultivadas, Instituto Brasileiro de Desenvolvimento Florestal: Rio de Janeiro, Brazil, 1984.

3. Souza, M. F; Rao, V. S.; Silveira, E. R.; Braz. J. Med. Biol. Res. 1992, 25, 1029.

4. Souza, M. F.; Cunha, G. M. A.; Fontenele, J. B.; Viana, G. S. B.; Rao, V. S.; Phytother. Res. 1994, 8, 478.

5. Melo, C.L.; Maia, S. B.; Souza, M. F.; Rao, V. S.; Lima, M. A. S.; Silveira, E. R.; Fitoterapia 1993, 64, 306. 
6. Rao, V. S.; Figueiredo, E. G.; Melo, C. L; Viana, G. S. B.; Menezes, D. B.; Matos, M. S. F.; Silveira, E. R. Pharmacology 1994, 48, 392.

7. Lima, M. A. S.; Silveira, E. R.; Marques, M. S. L.; Santos, R. H. A.; Gambardela, M. T. P.; Phytochemistry 1996, 41, 217.

8. Rao, V. S. N.; Paiva, L. A. F.; Souza, M. F.; Campos, A. R.; Ribeiro, R. A.; Brito, G. A. C. Souza, M. F.; Rao, V. S. N.; Silveira, E. R.; Phytomedicine 1997, 4, 27.

9. Rao, V. S. N.; Santos, F. A.; Sobreira, T. T.; Souza; M. F.; Melo, C. L.; Silveira, E. R.; Planta Med. 1997, 63, 146.

10. Souza, M. F.; Rao, V. S. N.; Silveira, E. R; Phytother. Res. 1998, $12,28$.

11. Guedes, M. M.; Cunha, A. N.; Silveira, E. R.; Rao, V. S. N.; Planta Med. 2002, 68, 1044.

12. Teixeira, M. J.; Silveira, E. R.; Planta Med. 2003, 69, 851.

13. Vieira, G. A. B.; Lima, M. A. S.; Bezerra, A. M.; Silveira, E. R.; J. Braz. Chem. Soc. 2006, 17, 43.

14. Melo, C. M.; Maia, J. L.; Cavalcante, I. J. M.; Lima, M. A. S.; Vieira, G. A. B.; Silveira, E. R.; Rao, V. S. N.; Santos, F. A.; Planta Med. 2006, 72, 584.

15. Lee, D.; Li, C.; Graf, T. N.; Vigo, J. S.; Graham, J. G.; Cabieses, F.; Farnsworth, N. R.; Cordell, G. A.; Kinghorn, A. D.; Kroll, D. J.; Wani, M. W.; Oberlies, N. H.; Planta Med. 2005, 71, 792.

16. Stierle, D. B.; Stierle, A. A.; Larsen, R. D.; Phytochemistry 1988 , $27,517$.
17. Rivero-Cruz, I.; Trejo, J. L.; Aguilar, M. I.; Bye, R.; Mata, R.; Planta Med. 2000, 66, 734.

18. Zhou, L.; Fuentes, R. R.; Hoffmann, J. J.; Timmermann, B. N.; Phytochemistry 1995, 40, 1201.

19. Henry, M.; Chatalat-Dublanche, I.; Planta Med. 1995, 4, 322.

20. Vieira, M. M.; Macedo, F. Y. B.; Belarmino Filho, J. N.; Costa, A. C. L. V.; Cunha, A. N.; Silveira, E. R.; Brito, G. A. C.; Ribeiro, R. A.; Phytother. Res. 2004, 18, 135.

21. Pessoa, C.; Silveira, E. R.; Lemos, T. L. G.; Wetmore, L. A.; Moraes, M. O.; Leyva, A.; Phytother. Res. 2000, 14, 187.

22. Souza, M. F.; Rao, V. S. N.; Silveira, E. R.; Phytother. Res. 1998 , $12,557$.

23. Souza, M. F.; Tome, A. R.; Rao, V. S. N.; Pharm. Pharmacol. 1999, 51, 125.

24. Breitmaier, E.; Voelter, N.; ${ }^{13}$ C NMR Spectroscopy Methods and Applications. Verlag Chimie: Düsseldorf, Germany, 1974.

25. Kouzi, S.A.; McChesney, J. D.; J. Nat. Prod. 1991, 54, 483.

26. Lee, S.O.; Choi, S. Z.; Choi, S. U.; Lee, K. C.; Chin, Y. W.; Kim, J.; Kim, Y. C.; Lee, K. R.; J. Nat. Prod. 2005, 68, 1471.

27. Torrenegra, R.; Robles, J.; Waibel, R.; Lowel, M.; Achenbach, H.; Phytochemistry 1994, 35, 195.

28. Mosmann, T.; J. Immunol. Methods 1983, 16, 55.

Received: May 25, 2007

Web Release Date: November 12, 2007 


\section{A Labdane Diterpene from the Aerial Parts of Egletes viscosa Less}

\section{Francisco Artur e Silva-Filho, ${ }^{a}$ Mary Anne S. Lima, ${ }^{* a}$ Antonio Marcos E. Bezerra, ${ }^{b}$ Raimundo Braz, Filho and Edilberto R. Silveira ${ }^{c}$ \\ ${ }^{a}$ Curso de Pós-Graduação em Química Orgânica, Universidade Federal do Ceará, CP 12.200 60.021-940, Fortaleza-CE, Brazil \\ ${ }^{b}$ Departamento de Fitotecnia, Universidade Federal do Ceará, CP 12168 60356-760 Fortaleza-CE, Brazil}

${ }^{c}$ Universidade Estadual do Norte Fluminense, Setor de Química de Produtos Naturais, 28013-600 Campos-RJ, Brazil

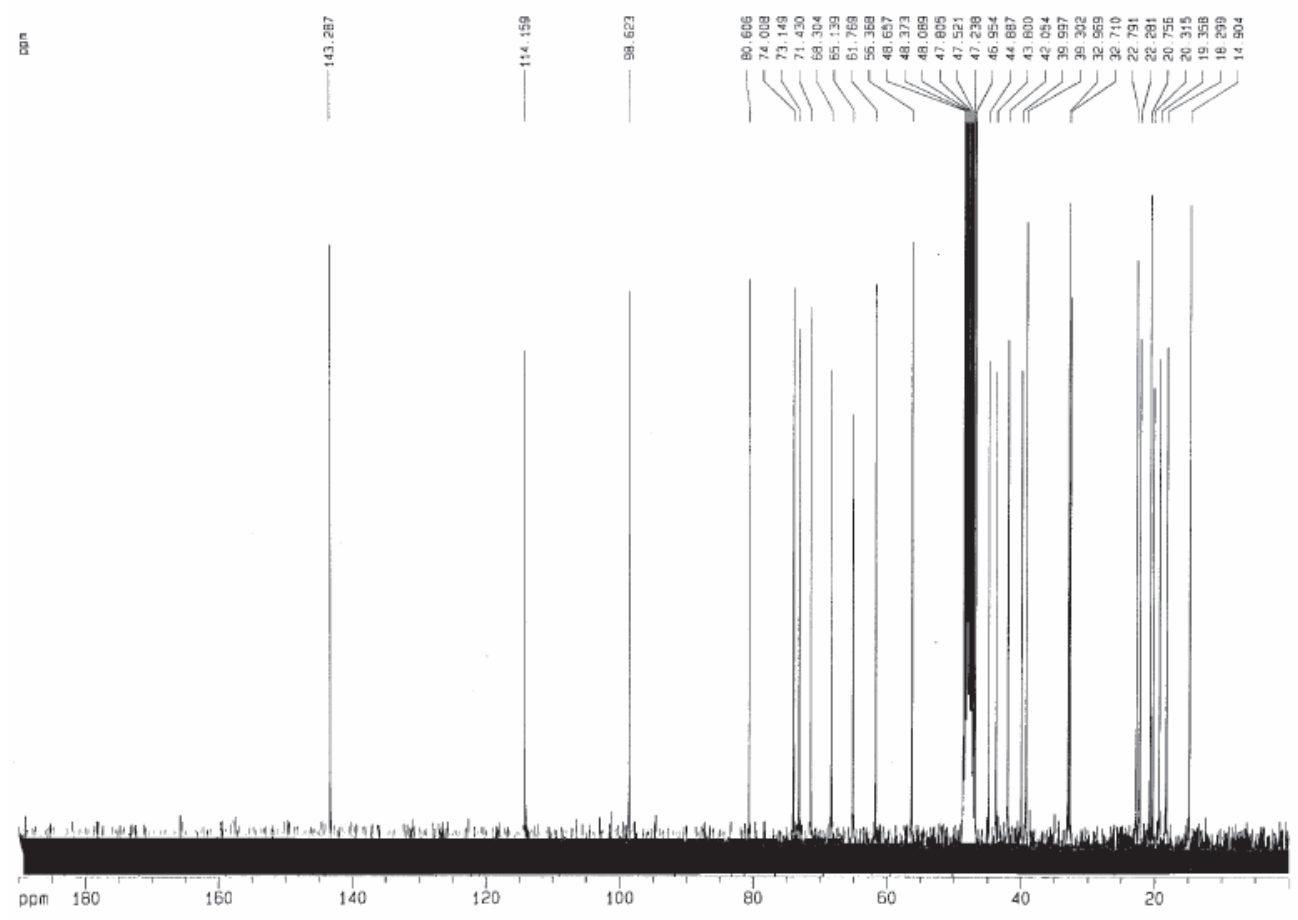

Figure S1. ${ }^{13} \mathrm{C}-\mathrm{BB}$ NMR spectrum (75 MHz, MeOD) of 1 (8 $\alpha$-hydroxylabd-14(15)-ene-13(S)-O- $\beta$-D-ribopyranoside).

*e-mail: mary@dqoi.ufc.br 


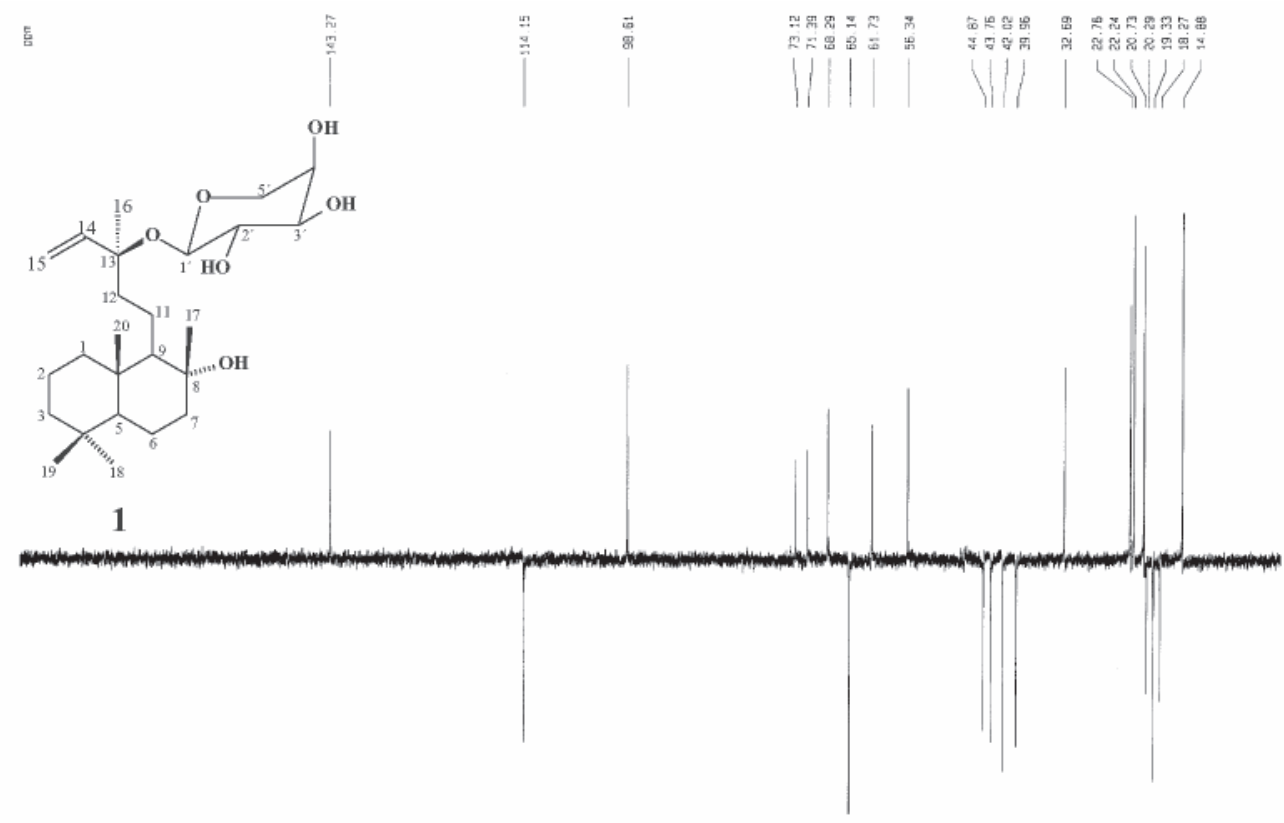

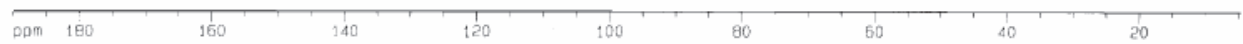

Figure S2. ${ }^{13} \mathrm{C}$-DEPT $135^{\circ} \mathrm{NMR}$ spectrum (75 MHz, MeOD) of 1 (8 $\alpha$-hydroxylabd-14(15)-ene-13(S)-O- $\beta$-D-ribopyranoside).
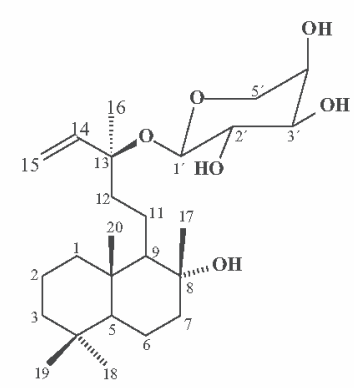

1

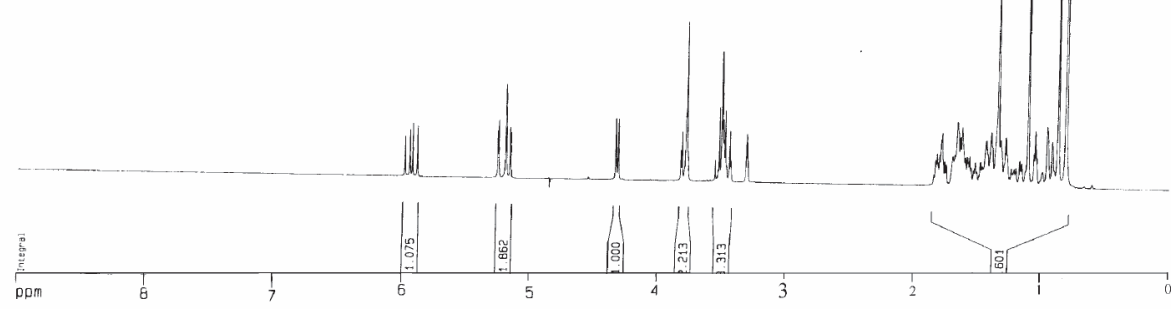

Figure S3. ${ }^{1} \mathrm{H}$ NMR spectrum (300 MHz, MeOD) of 1 (8 $\alpha$-hydroxylabd-14(15)-ene-13(S)-O- $\beta$-D-ribopyranoside). 


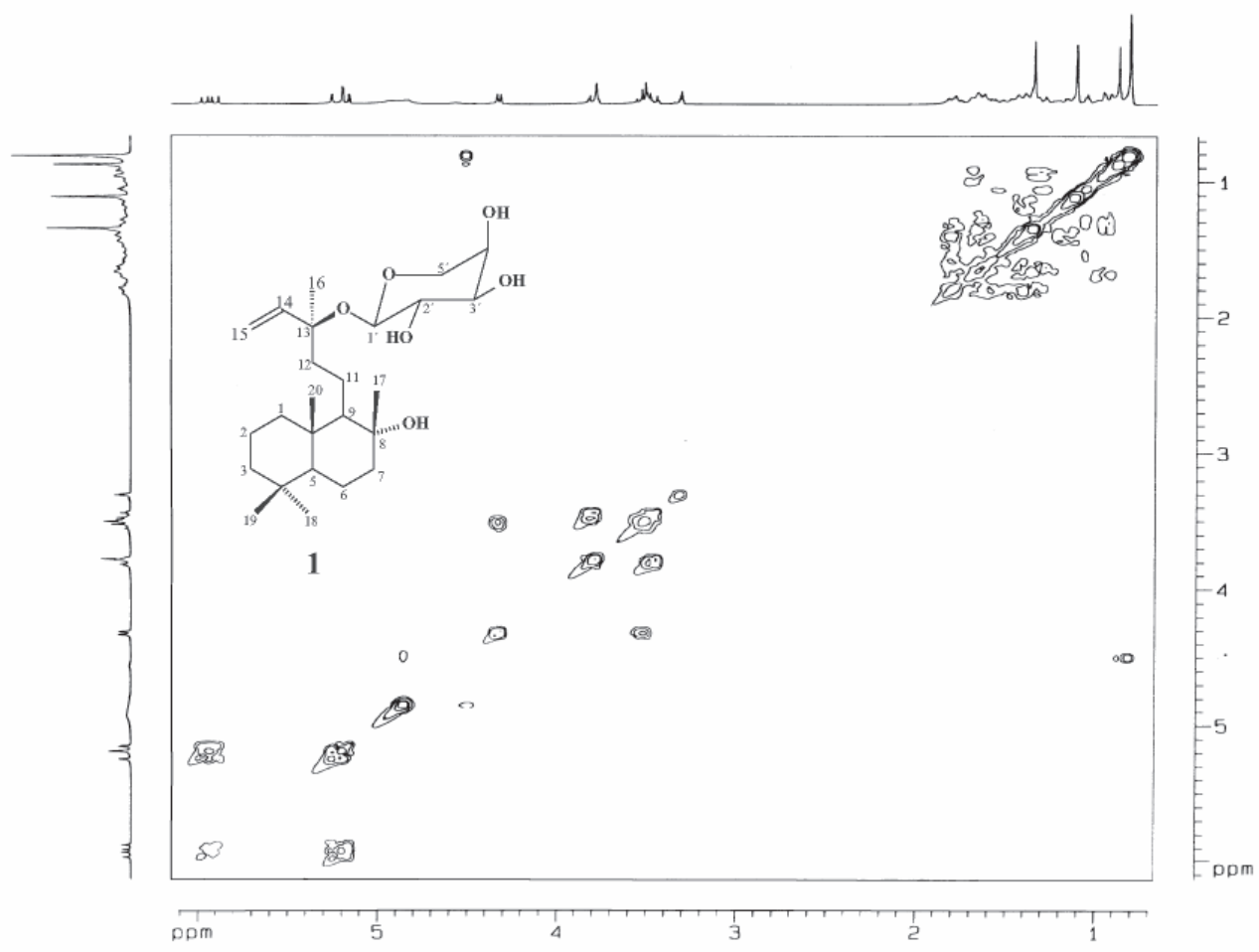

Figure S4. COSY NMR spectrum (300 MHz, MeOD) of 1 (8 $\alpha$-hydroxylabd-14(15)-ene-13(S)-O- $\beta$-D-ribopyranoside).

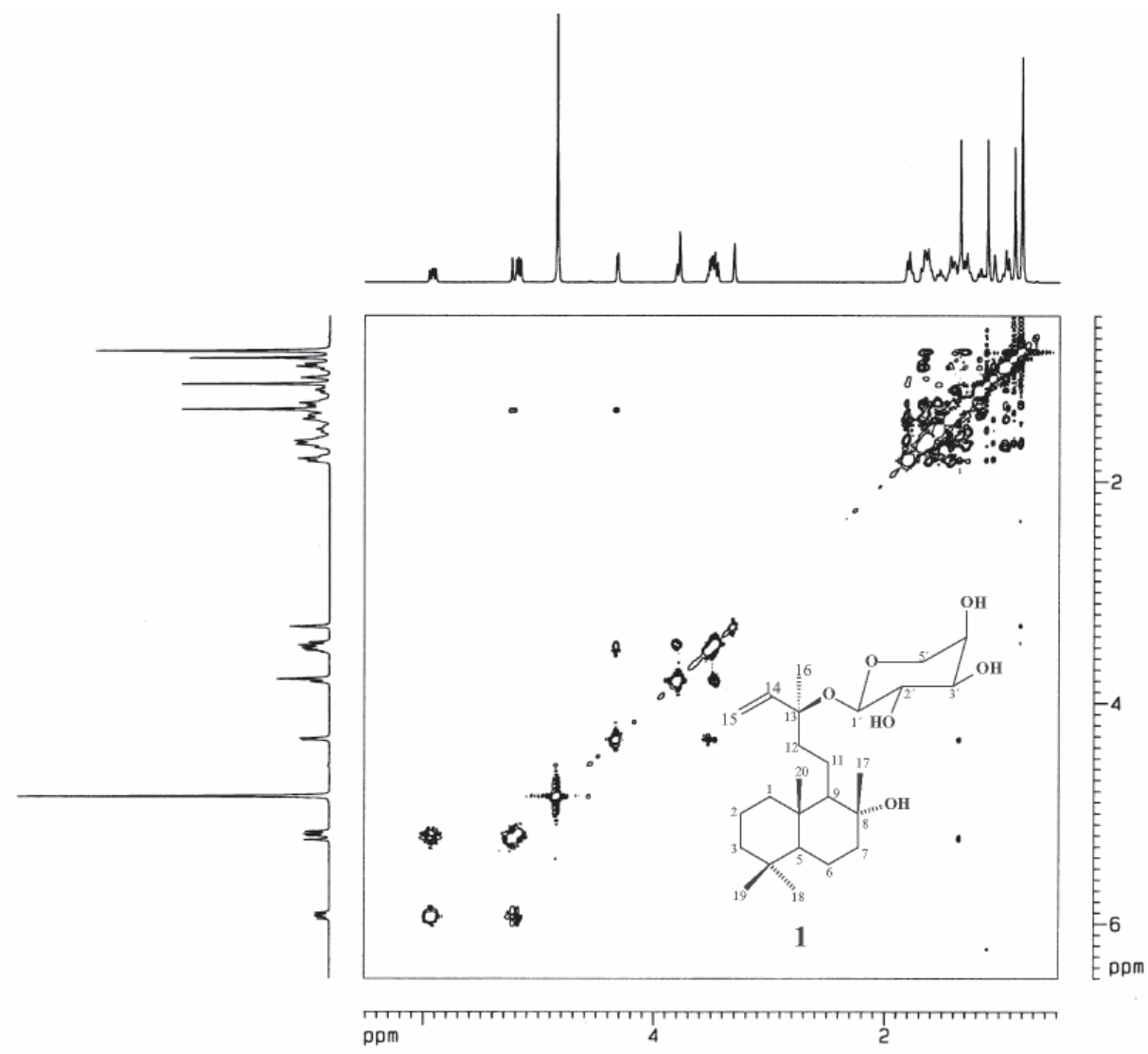

Figure S5. NOESY NMR spectrum (300 MHz, MeOD) of 1 ( $8 \alpha$-hydroxylabd-14(15)-ene-13(S)-O- $\beta$-D-ribopyranoside). 


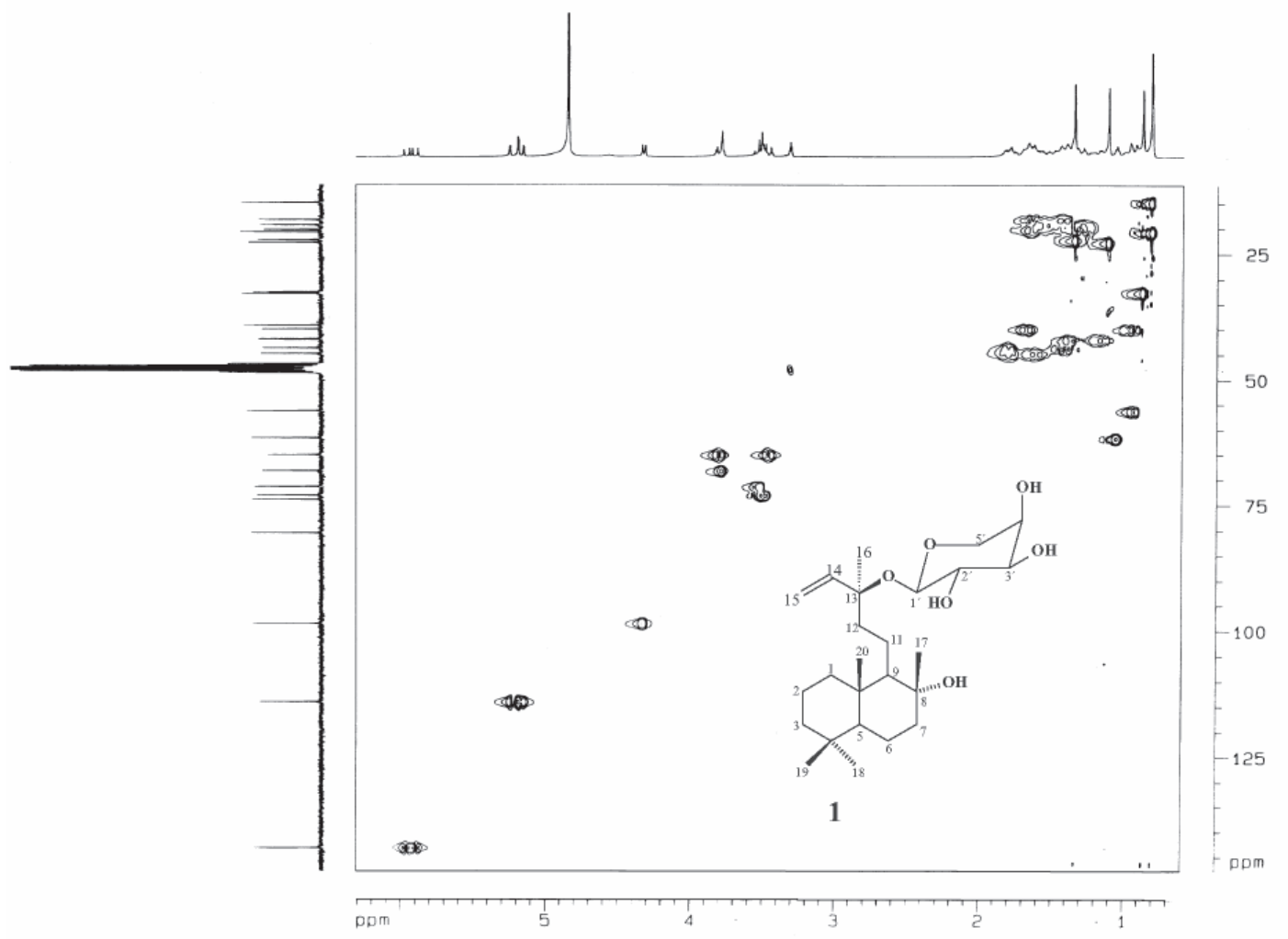

Figure S6. HMQC NMR spectrum (300 MHz, MeOD) of 1 (8 $\alpha$-hydroxylabd-14(15)-ene-13(S)-O- $\beta$-D-ribopyranoside).

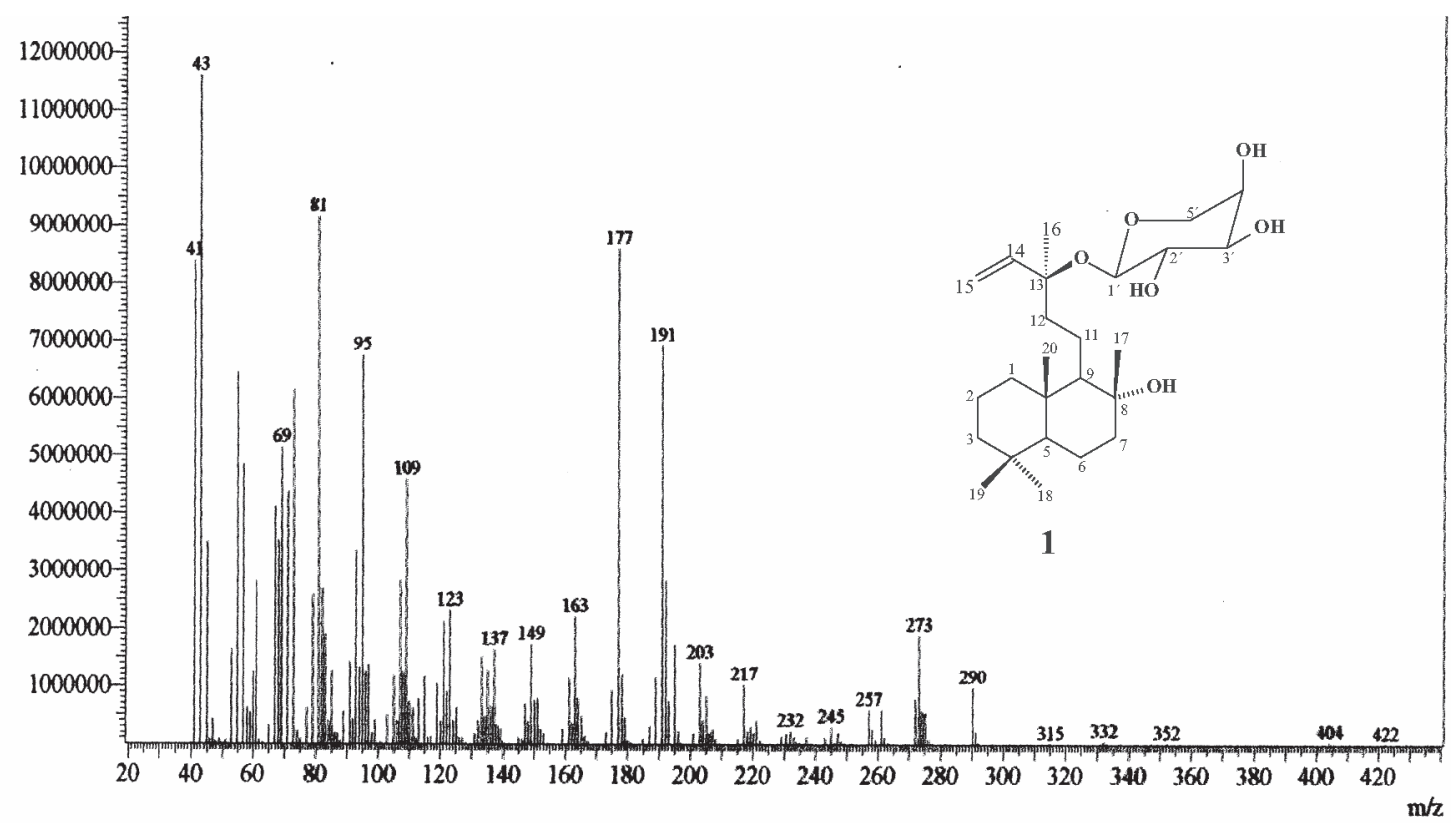

Figure S7. HR-EIMS spectrum of 1 ( $8 \alpha$-hydroxylabd-14(15)-ene-13(S)-O- $\beta$-D-ribopyranoside). 

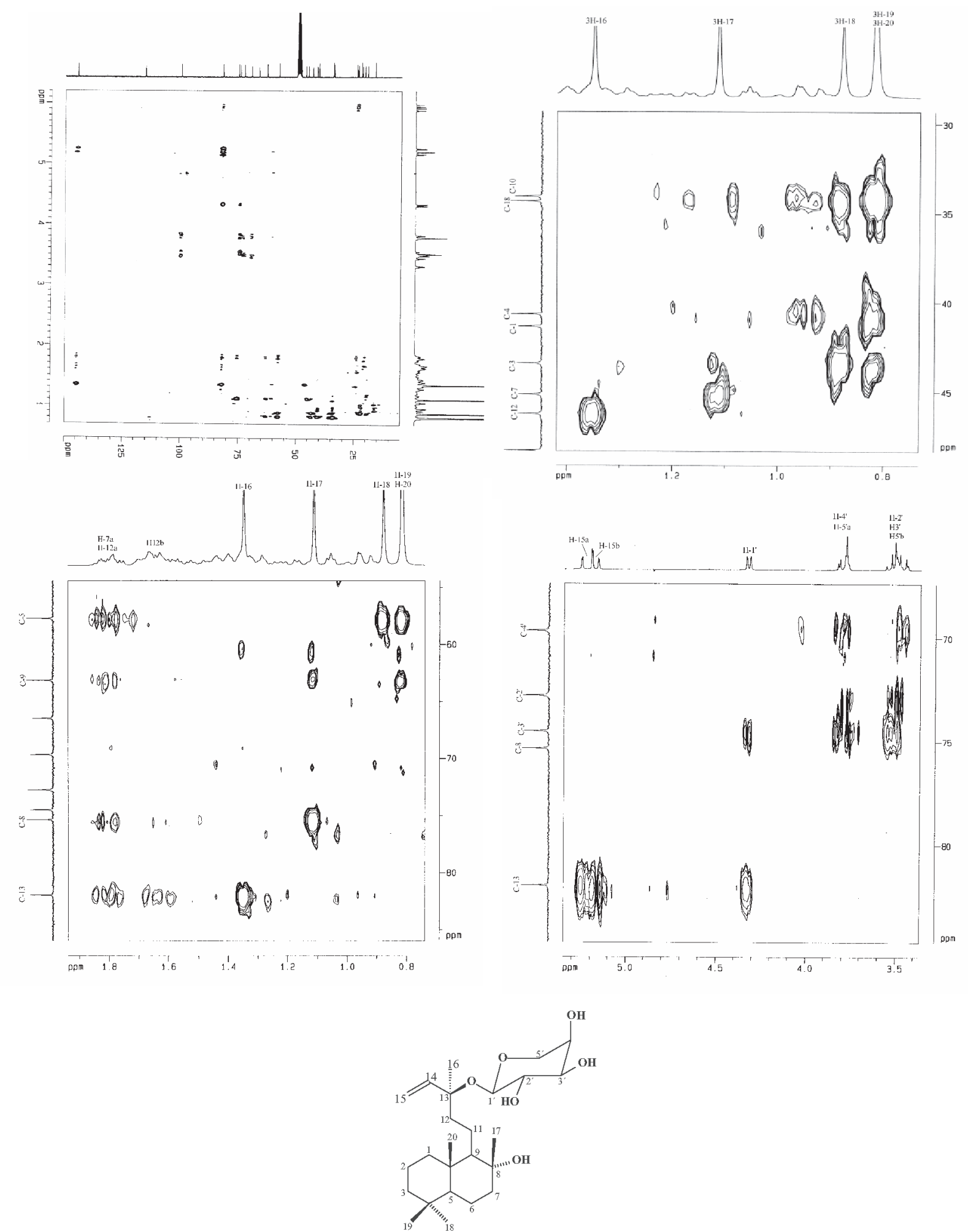

1

Figure S8. HMBC NMR and expansion spectrum (300 MHz, MeOD) of 1 (8 $\alpha$-hydroxylabd-14(15)-ene-13(S)-O- $\beta$-D-ribopyranoside) 
흘
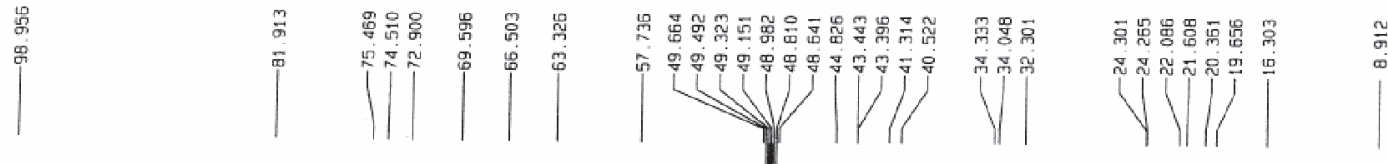

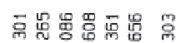<smiles>CCC(CC)OCC(C)COCC(O)CO</smiles><smiles>CCC1C2CCCC(C)(C)C2CCC1(O)F</smiles>

2

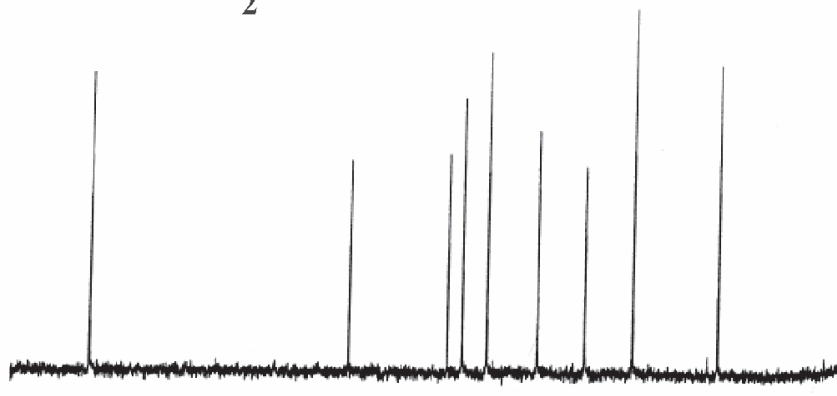

Dom

80

60

10

20

Figure S9. ${ }^{13} \mathrm{C}-\mathrm{BB}$ NMR spectrum (125 MHz, MeOH) of 2 ( $8 \alpha$-hydroxylabd-13(S)-O- $\beta$-D-ribopyranoside).

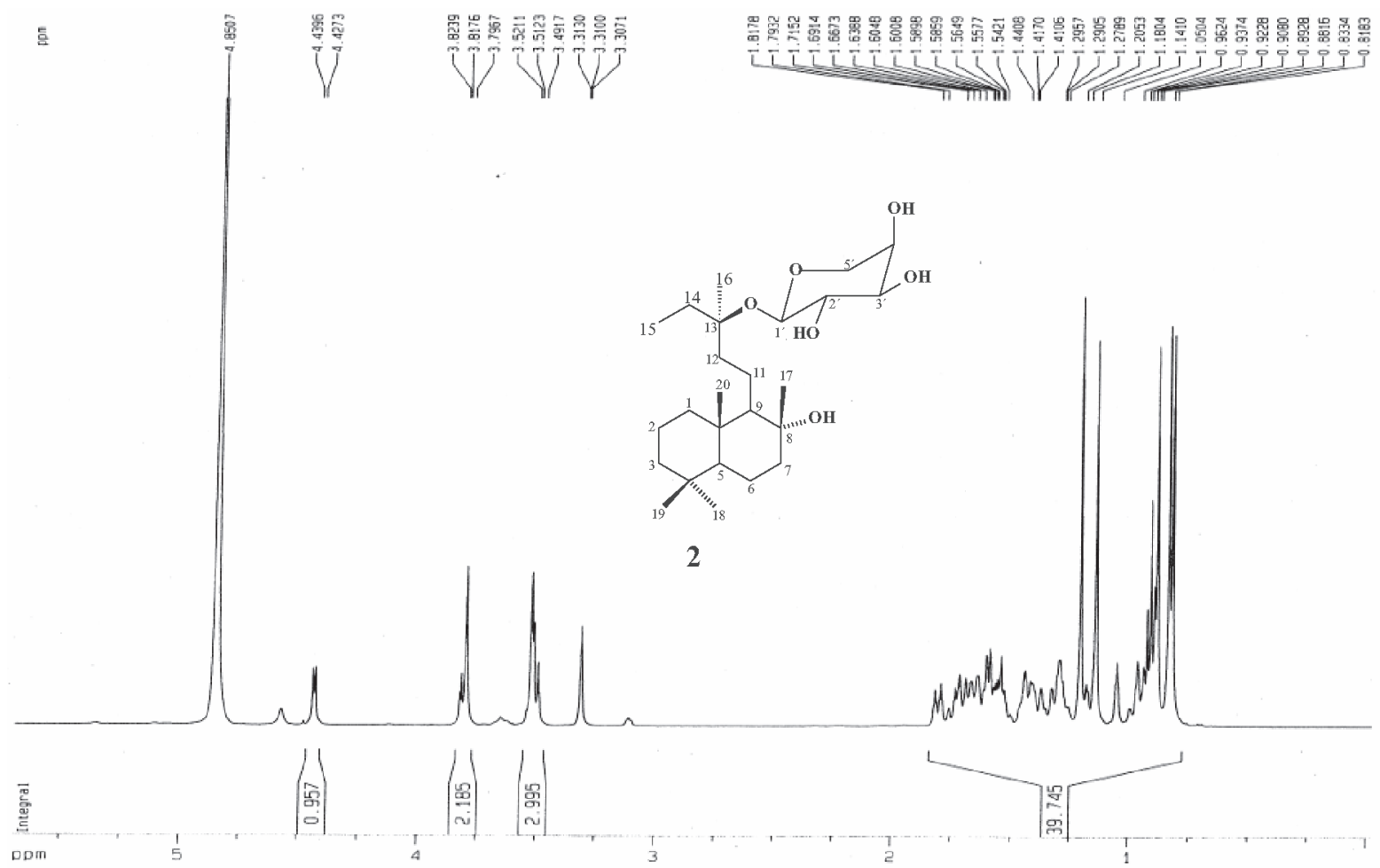

Figure S10. ${ }^{1} \mathrm{H}$ NMR spectrum (500 MHz, MeOH) of 2 (8 $\alpha$-hydroxylabd-13(S)-O- $\beta$-D-ribopyranoside). 


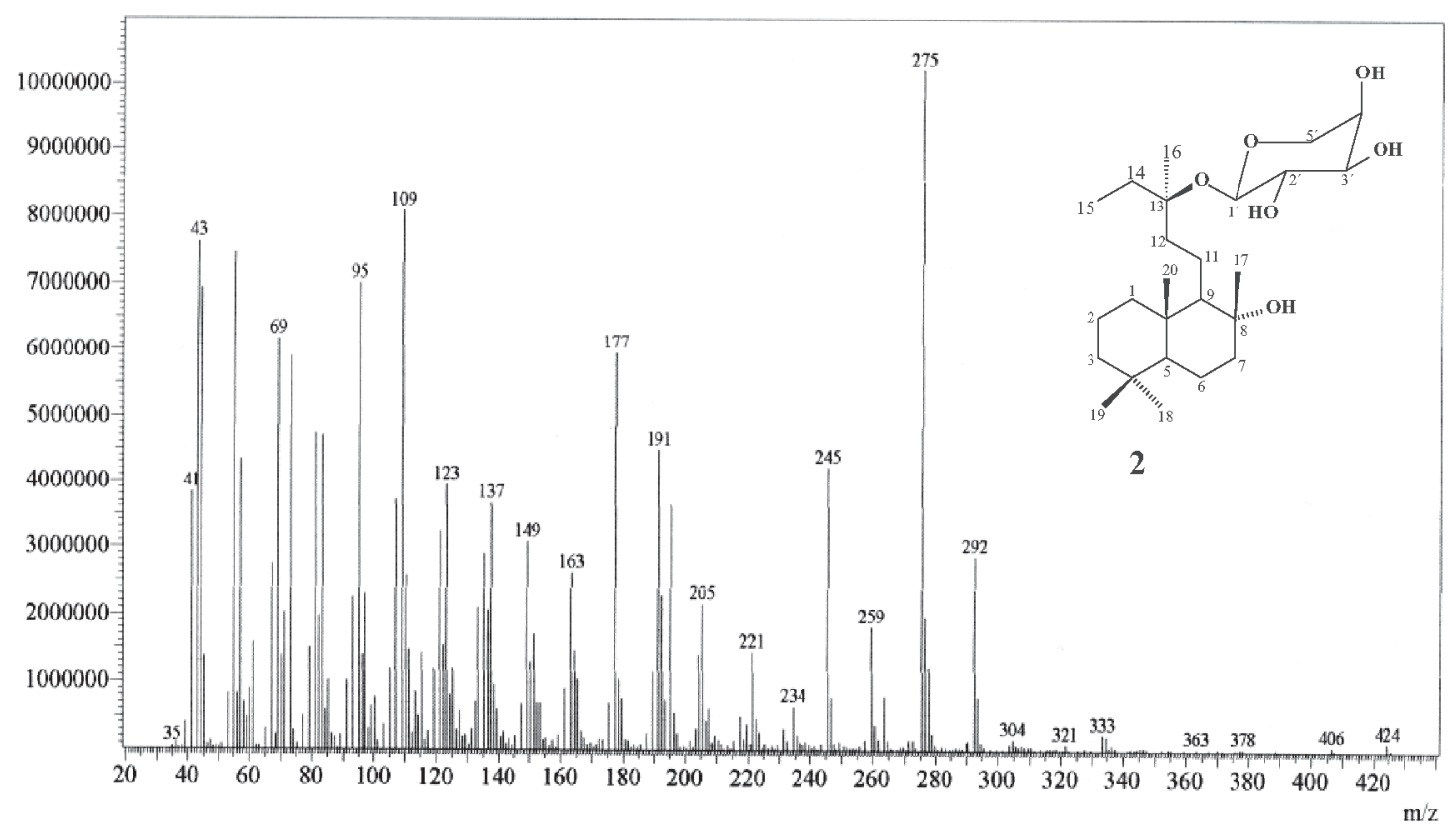

Figure S11. HR-EIMS spectrum of 2 (8 $\alpha$-hydroxylabd-13(S)-O- $\beta$-D-ribopyranoside).

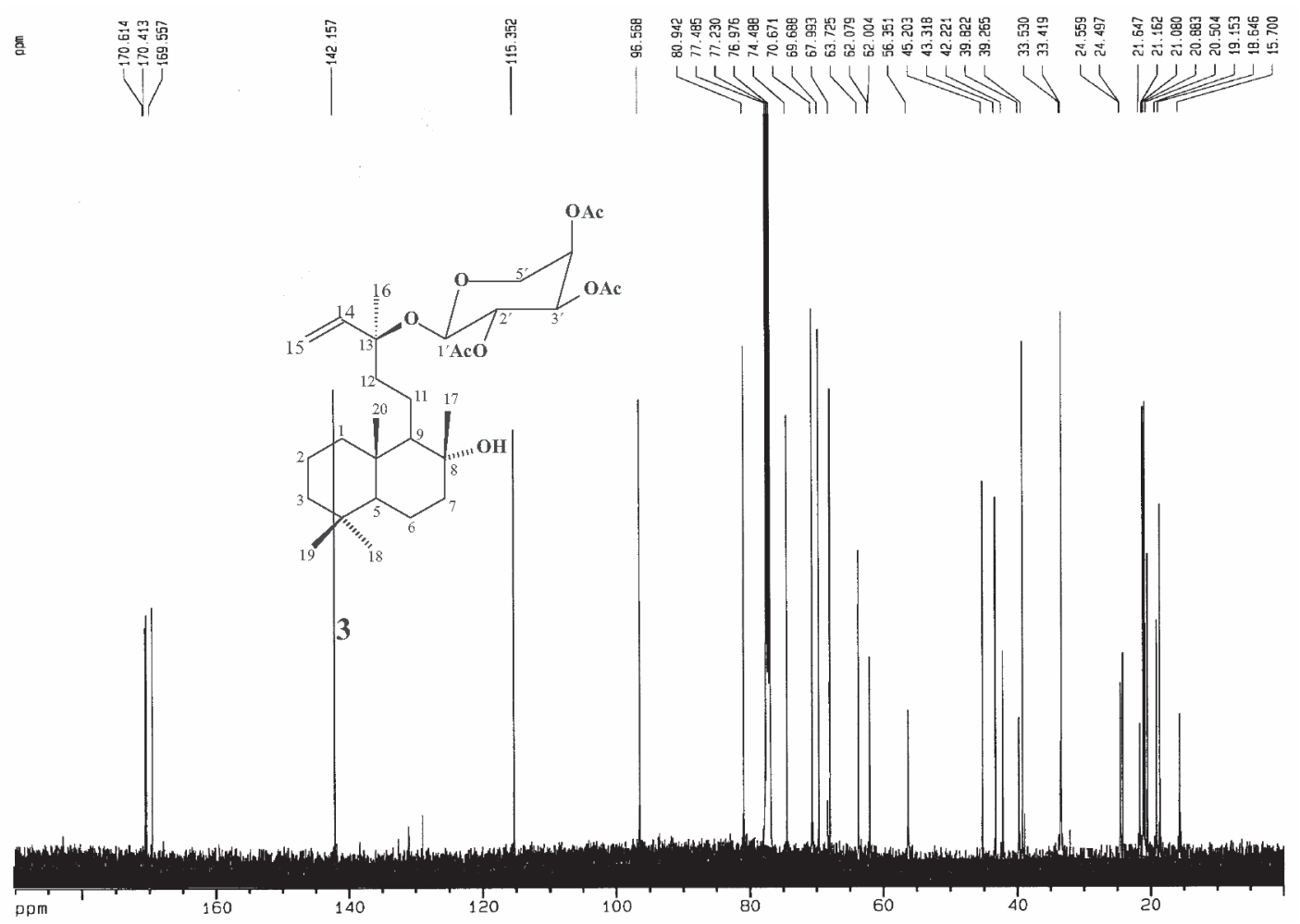

Figure S12. ${ }^{13} \mathrm{C}-\mathrm{BB}$ NMR spectrum (125 MHz, $\mathrm{CDCl}_{3}$ ) of 3 ( $8 \alpha$-hydroxylabd-14(15)-ene-13(S)-O- $\beta$-D-2', $3^{\prime}, 4^{\prime}$ - triacetylribopyranoside). 

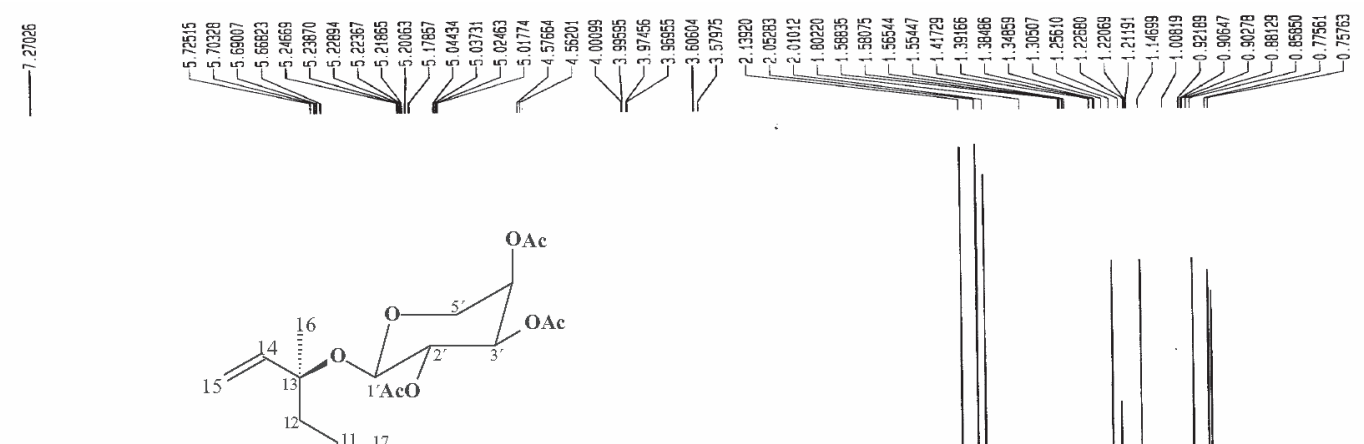<smiles>CC1CCC(C)(C)C2CC[C@](C)(O)C(I)C12</smiles>

3

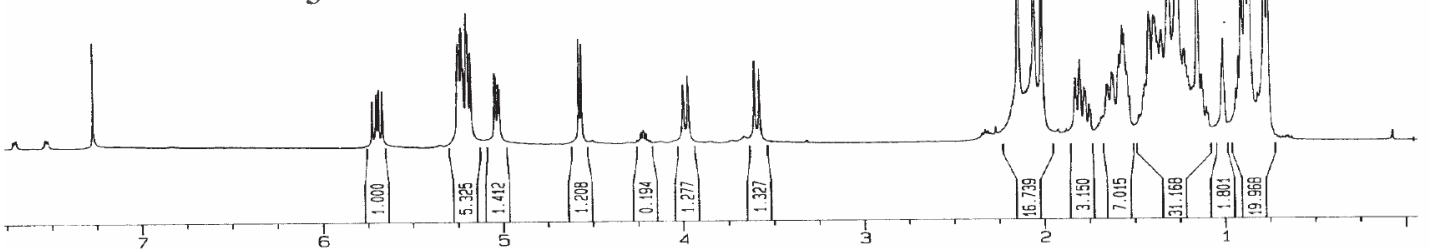

Figure S13. ${ }^{~} \mathrm{H}$ NMR spectrum ( $500 \mathrm{MHz}, \mathrm{CDCl}_{3}$ ) of 3 (8 $\alpha$-hydroxylabd-14(15)-ene-13(S)-O- $\beta$-D-2', $3^{\prime}, 4^{\prime}$ - triacetylribopyranoside).

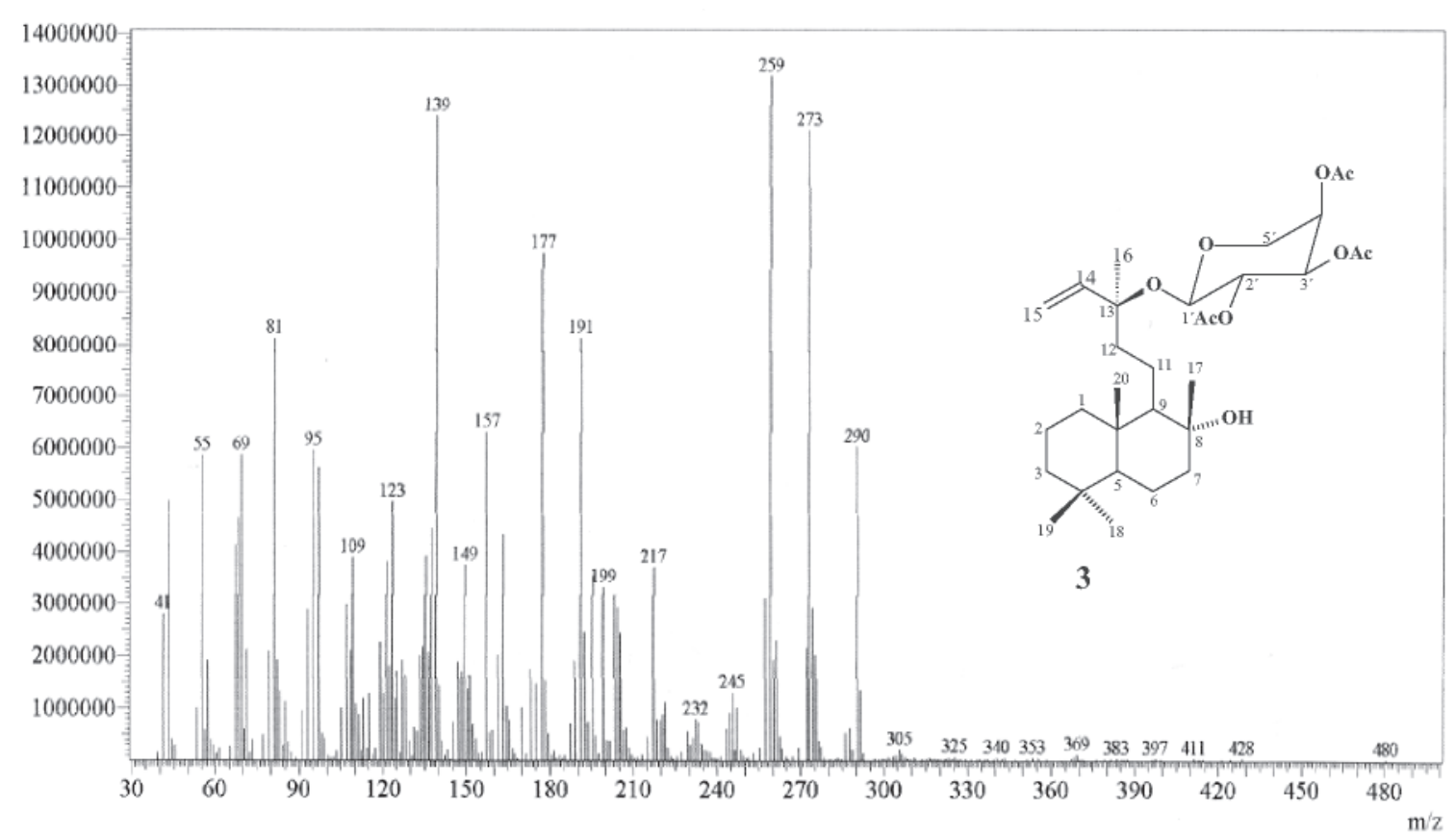

Figure S14. HR-EIMS spectrum of 3 (8 $\alpha$-hydroxylabd-14(15)-ene-13(S)-O- $\beta$-D-2', 3', $4^{\prime}$ - triacetylribopyranoside). 

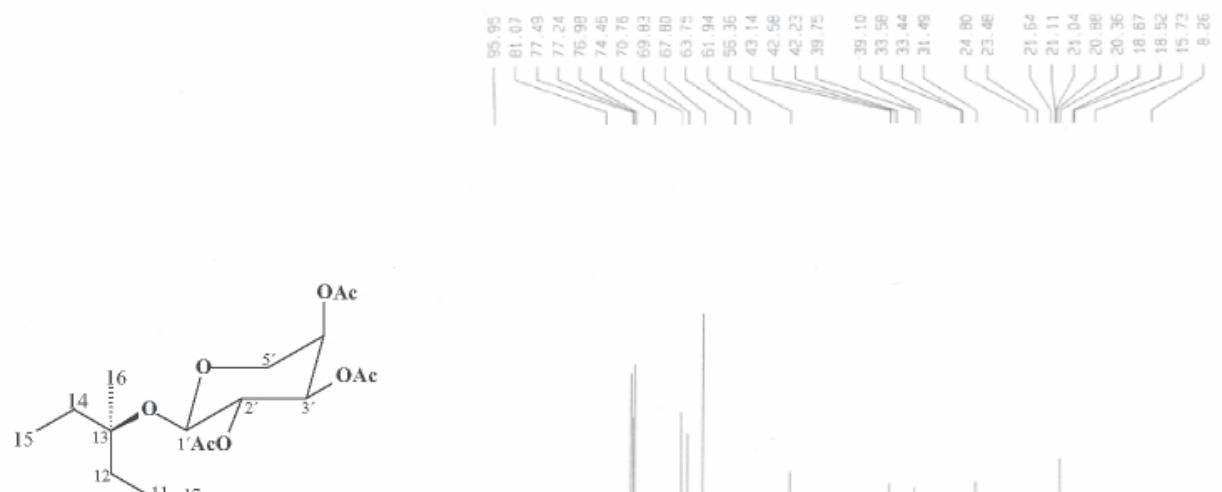<smiles>CC1CCCCC1(C)[C@@H](C)O</smiles>

4

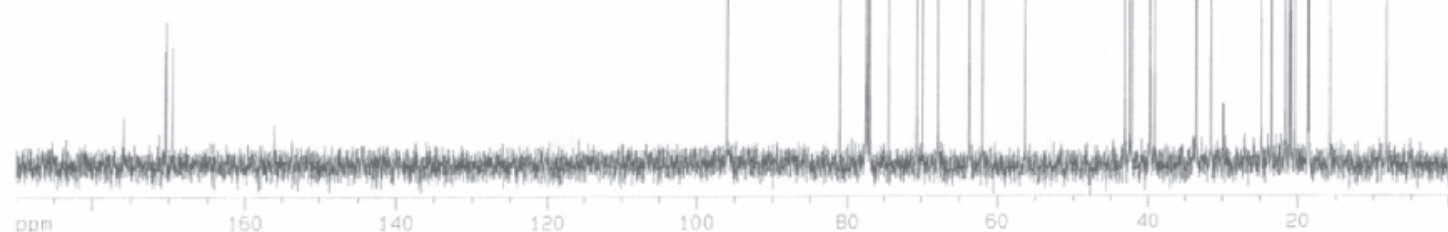

Figure S15. ${ }^{13} \mathrm{C}-\mathrm{BB}$ NMR spectrum (125 MHz,CDCl 3 ) of 4 ( $8 \alpha$-hydroxylabd-13(S)-O- $\beta$-D-2' $3^{\prime}, 4^{\prime}$-triacetylribopyranoside).

흥

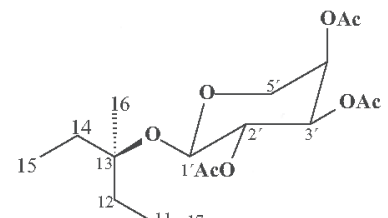<smiles>CCC1C(O)(F)CCC2C(C)(C)CCCC21C</smiles>

4

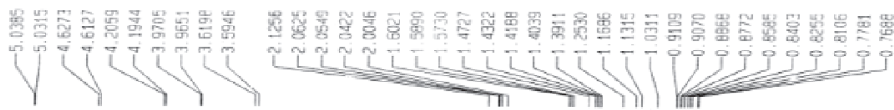

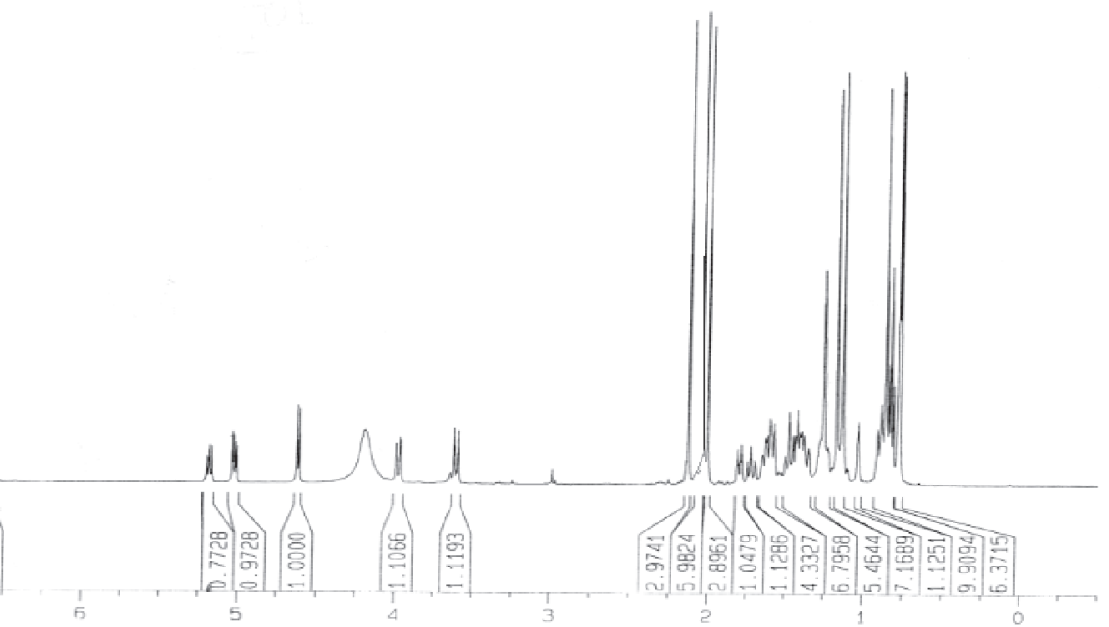

Figure S16. ${ }^{1} \mathrm{H}$ NMR spectrum ( $500 \mathrm{MHz}, \mathrm{CDCl}_{3}$ ) of 4 ( $8 \alpha$-hydroxylabd-13(S)-O- $\beta$-D-2', 3', 4' -triacetylribopyranoside). 


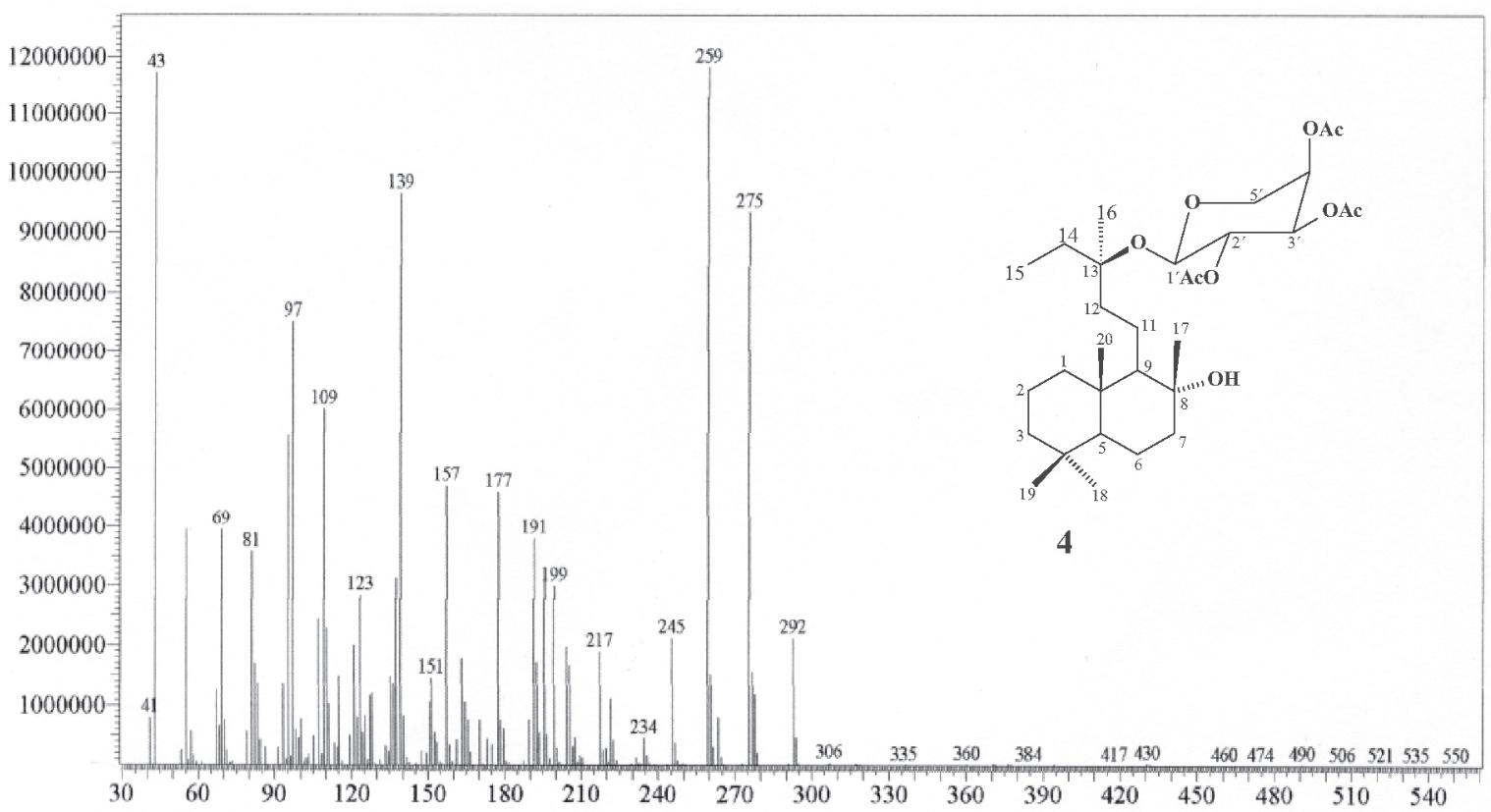

Figure S17. HR-EIMS spectrum of 4 ( $8 \alpha$-hydroxylabd-13(S)-O- $\beta$-D-2', $3^{\prime}, 4^{\prime}$-triacetylribopyranoside).

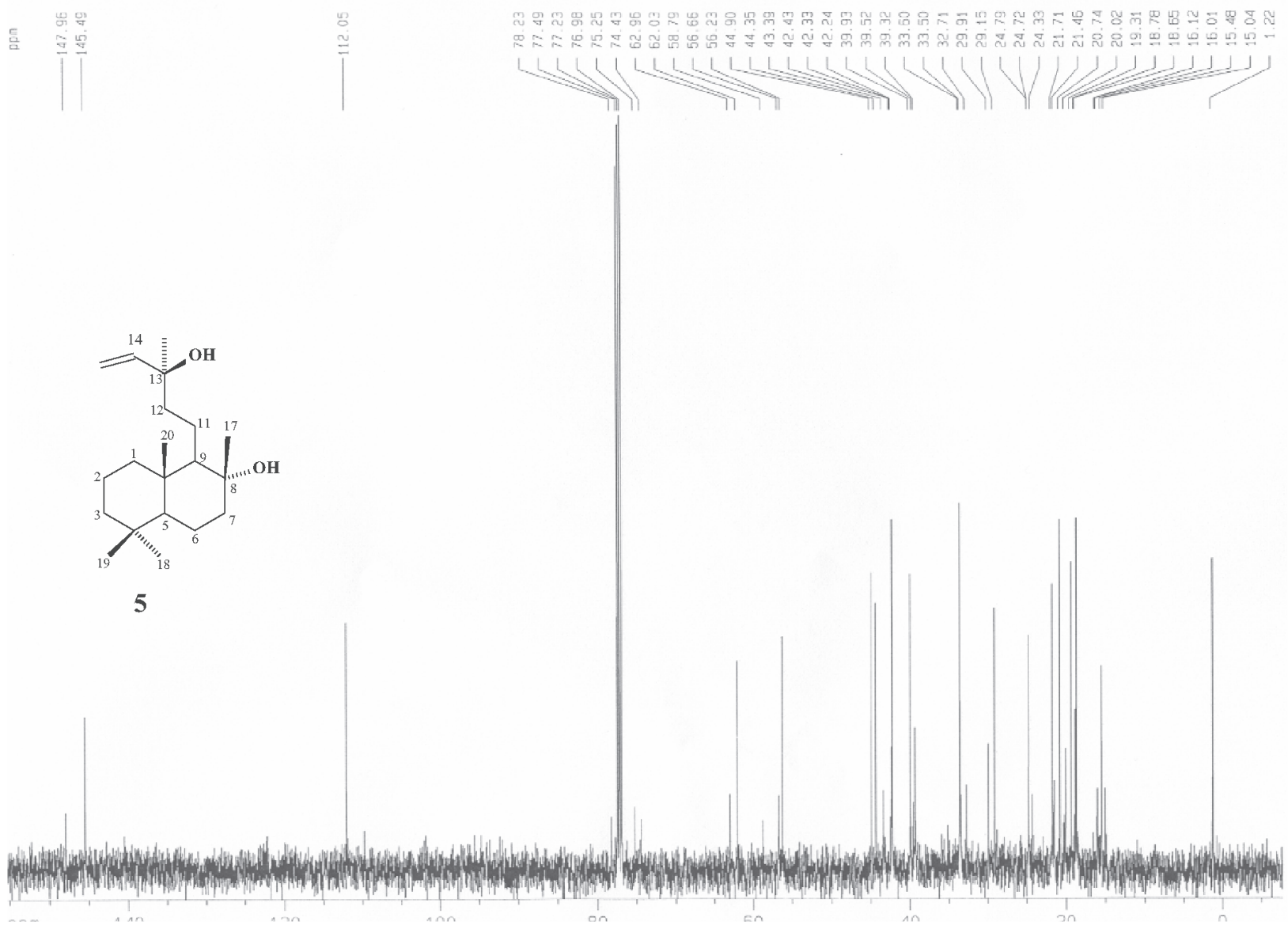

Figure S18. ${ }^{13} \mathrm{C}-\mathrm{BB}$ NMR spectrum $\left(125 \mathrm{MHz}, \mathrm{CDCl}_{3}\right)$ of 5 (13-epi-sclareol). 
气
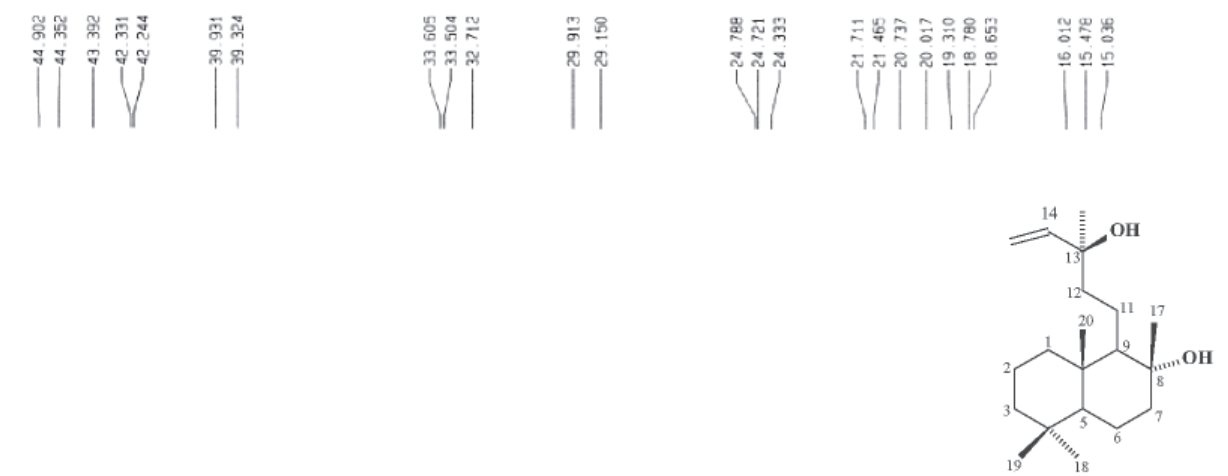

5

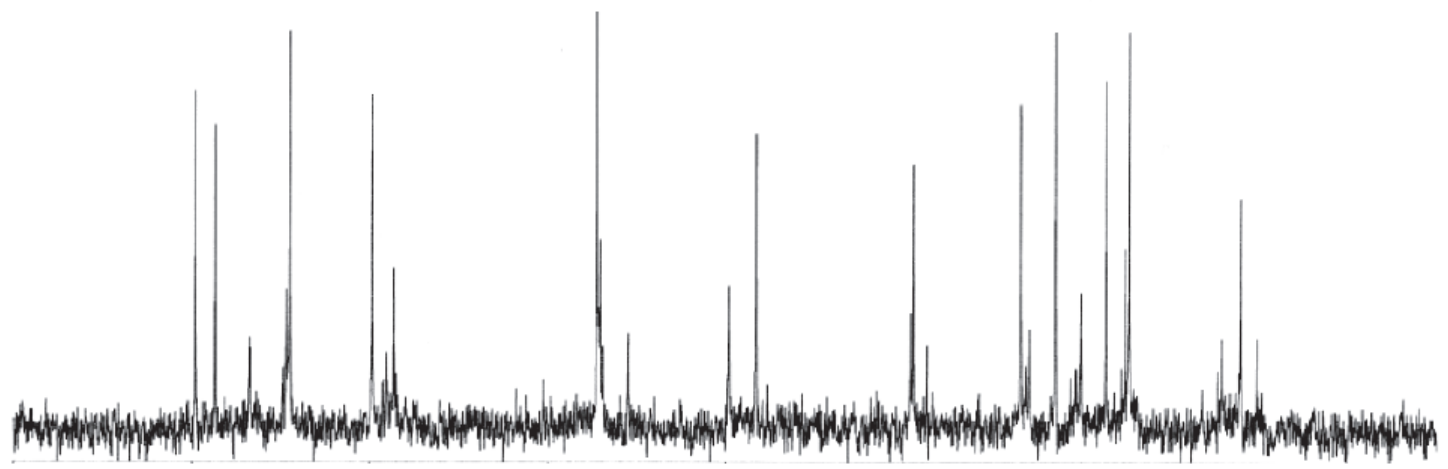

Figure S19. ${ }^{13} \mathrm{C}$-BB NMR expansion spectrum $\left(125 \mathrm{MHz}, \mathrm{CDCl}_{3}\right.$ ) of $\mathbf{5}$ (13-epi-sclareol).
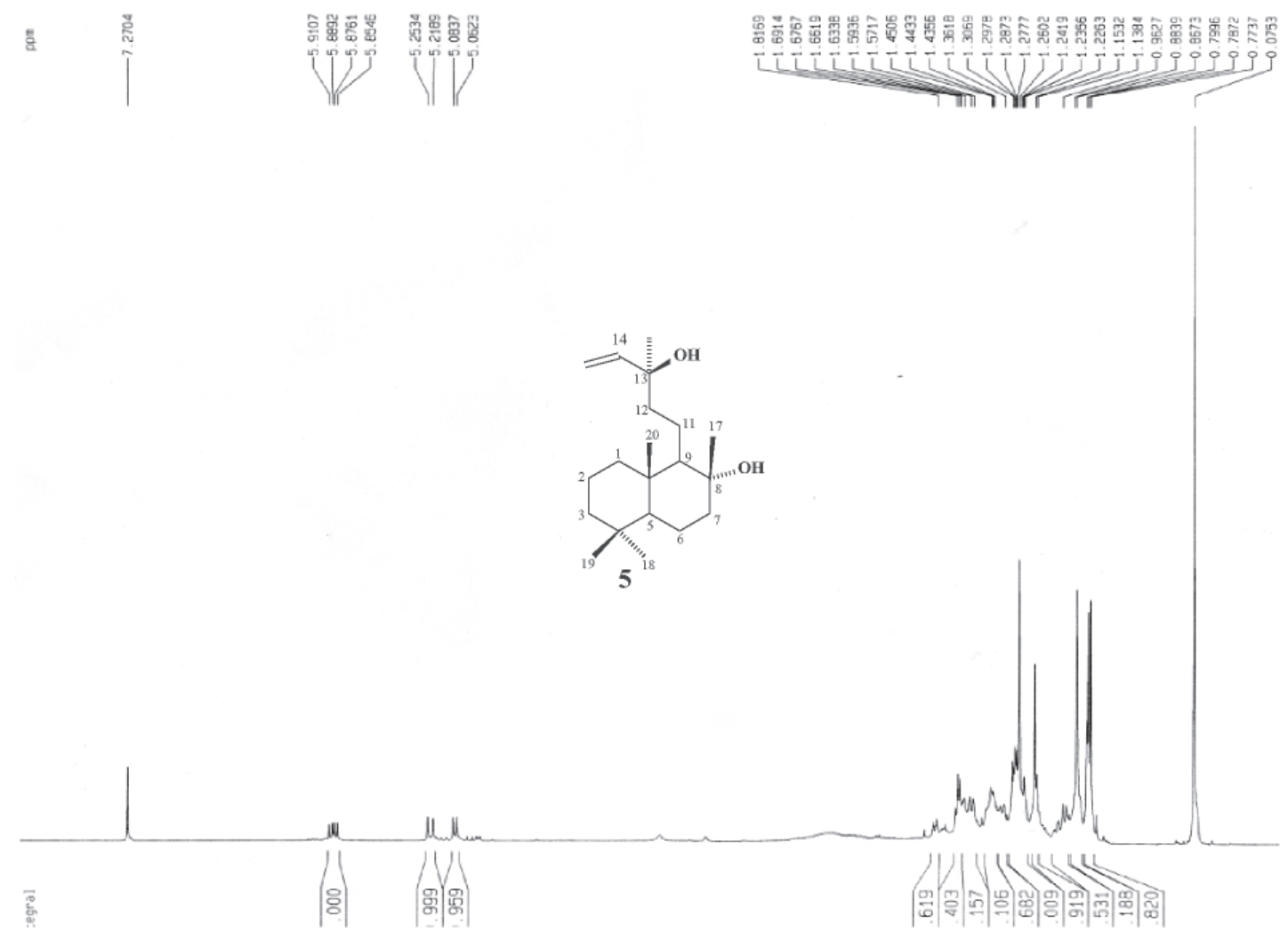

Figure S20. ${ }^{1} \mathrm{H}$ NMR spectrum $\left(500 \mathrm{MHz}, \mathrm{CDCl}_{3}\right.$ ) of 5 (13-epi-sclareol). 


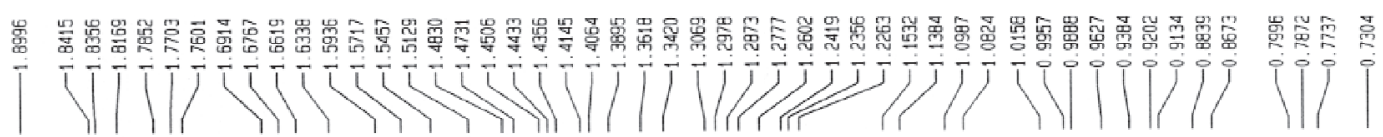<smiles>C=C[C@H](O)CCC1[C@H]2CCCC(C)(C)[C@@H]2CC[C@@]1(C)O</smiles>

5

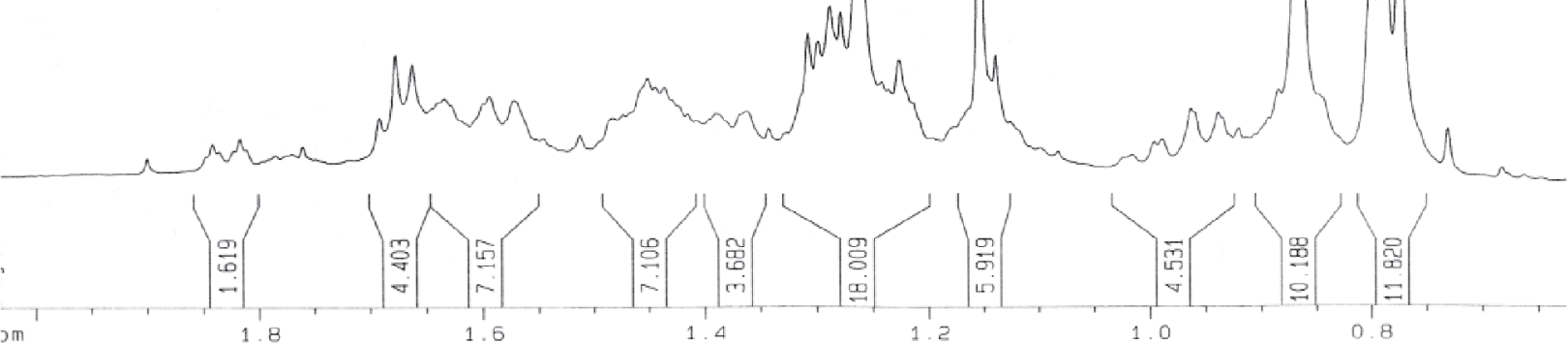

Figure S21. ' $\mathrm{H}$ NMR expansion spectrum ( $500 \mathrm{MHz}, \mathrm{CDCl}_{3}$ ) of 5 (13-epi-sclareol).

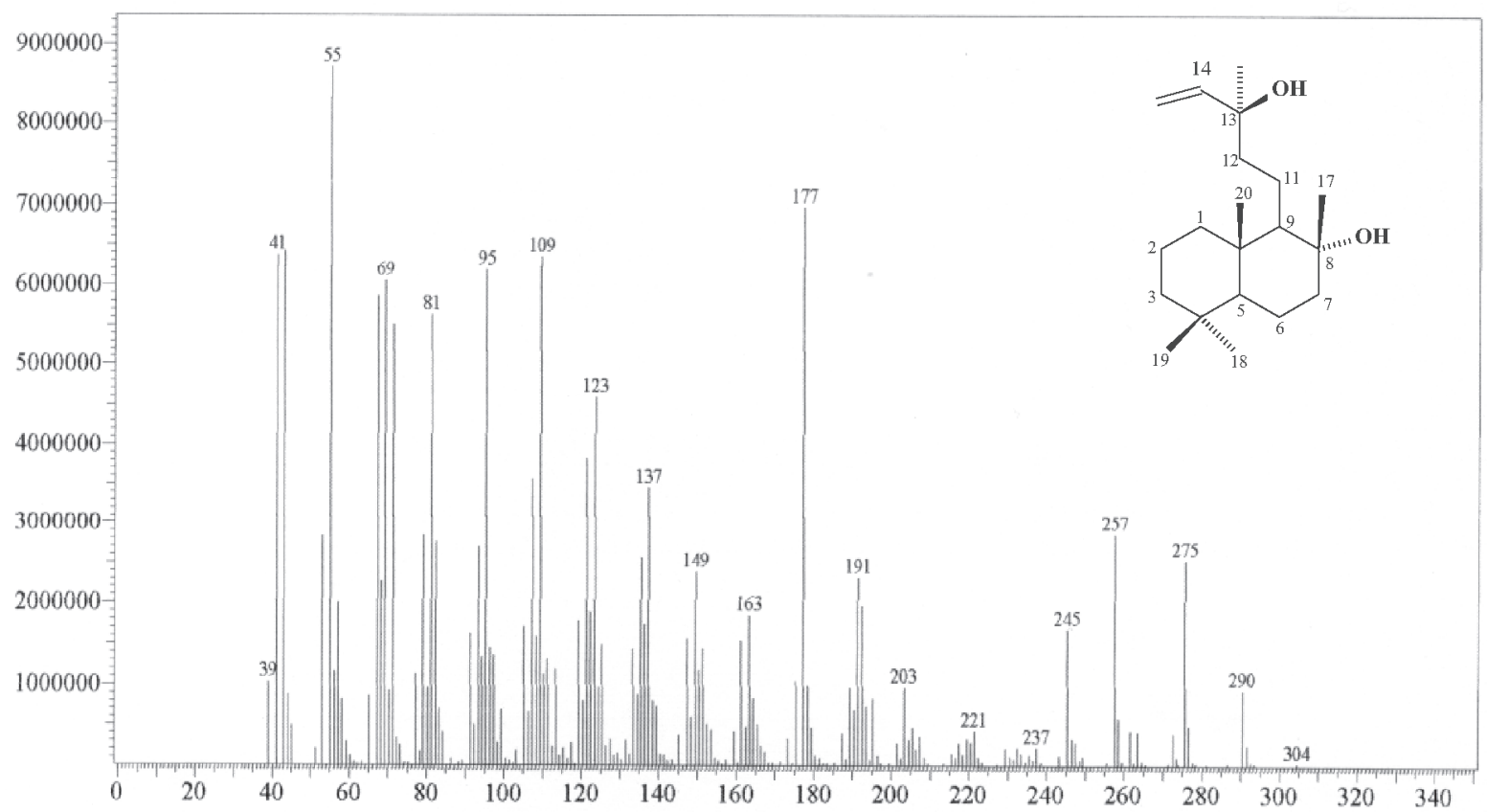

Figure S22. HR-EIMS spectrum of 5 (13-epi-sclareol). 


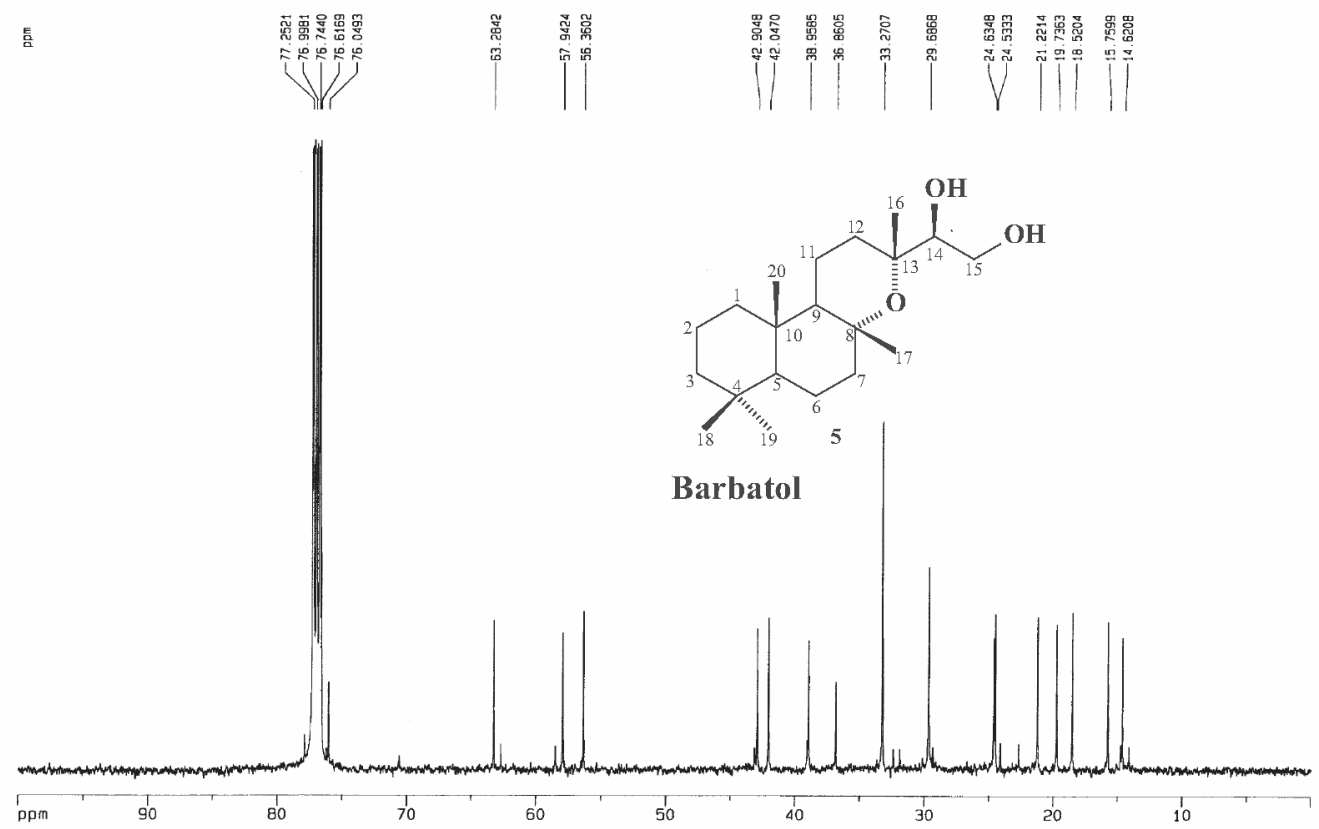

Figure S23. ${ }^{13} \mathrm{C}-\mathrm{BB}$ NMR spectrum $\left(125 \mathrm{MHz}, \mathrm{CDCl}_{3}\right)$ of Barbatol.
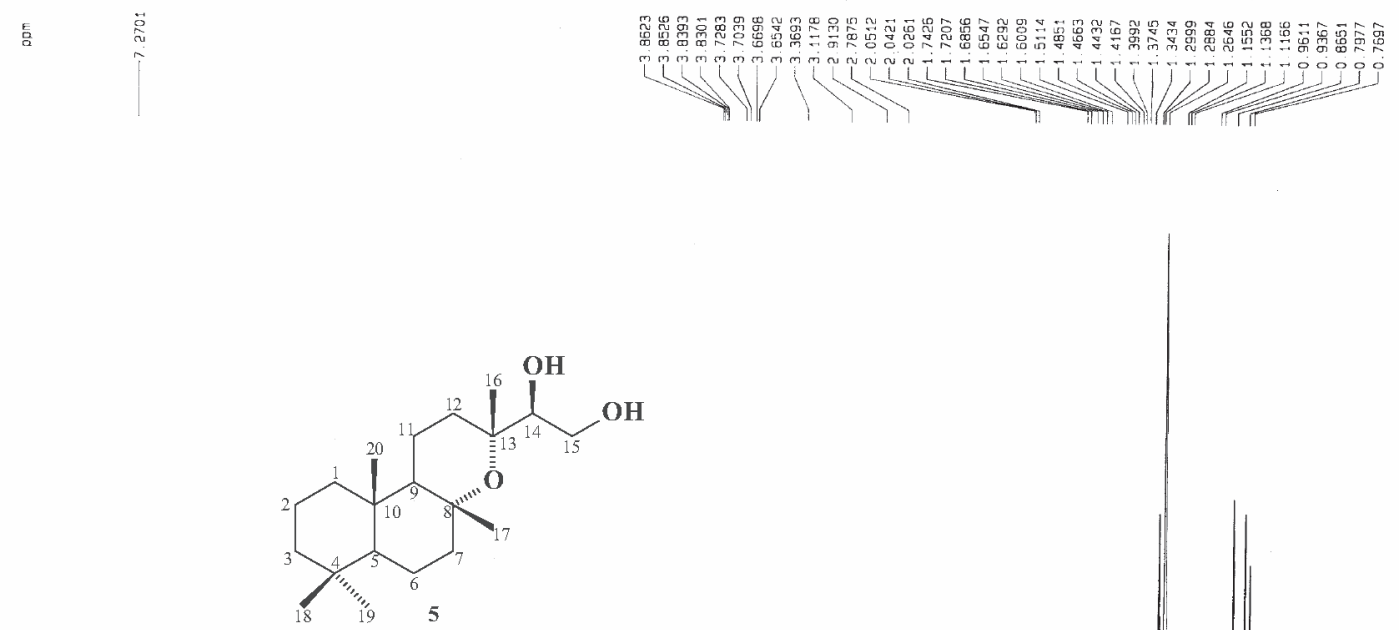

Barbatol

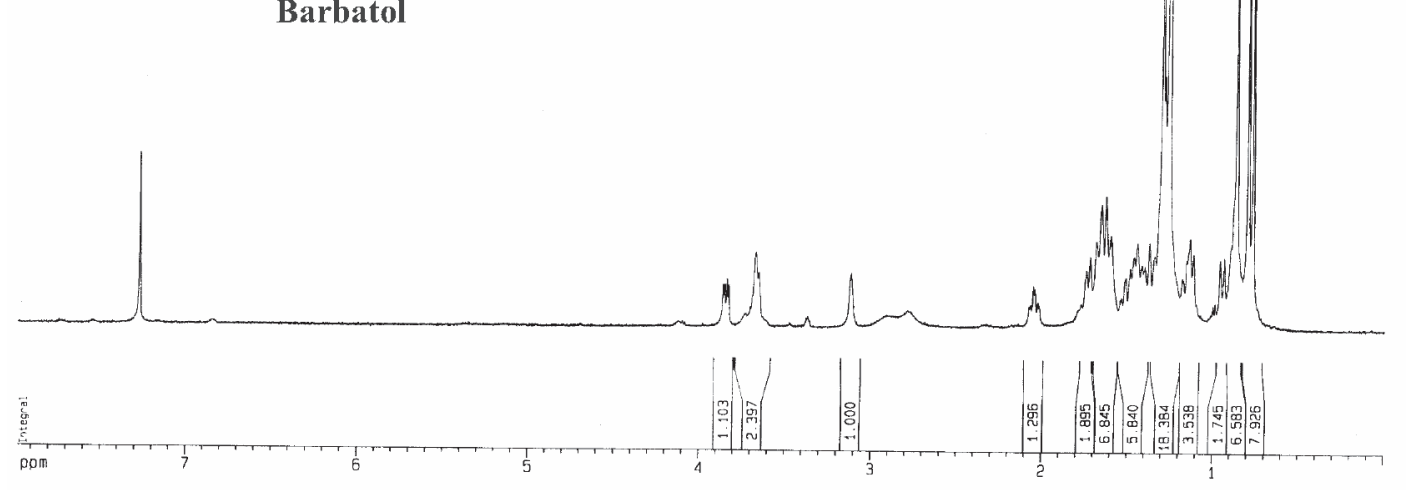

Figure S24. ${ }^{1} \mathrm{H}$ NMR spectrum $\left(500 \mathrm{MHz}, \mathrm{CDCl}_{3}\right)$ of Barbatol. 


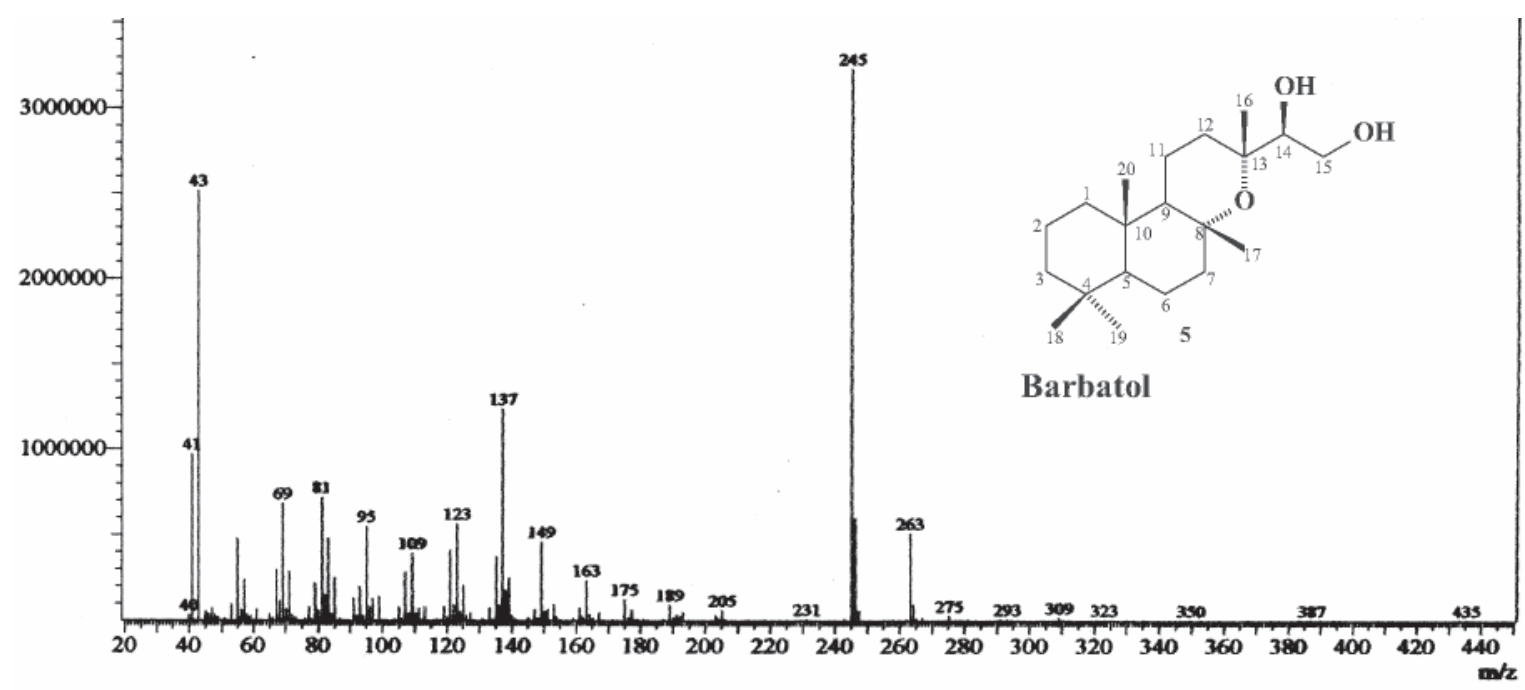

Figure S25. HR-EIMS spectrum of Barbatol.

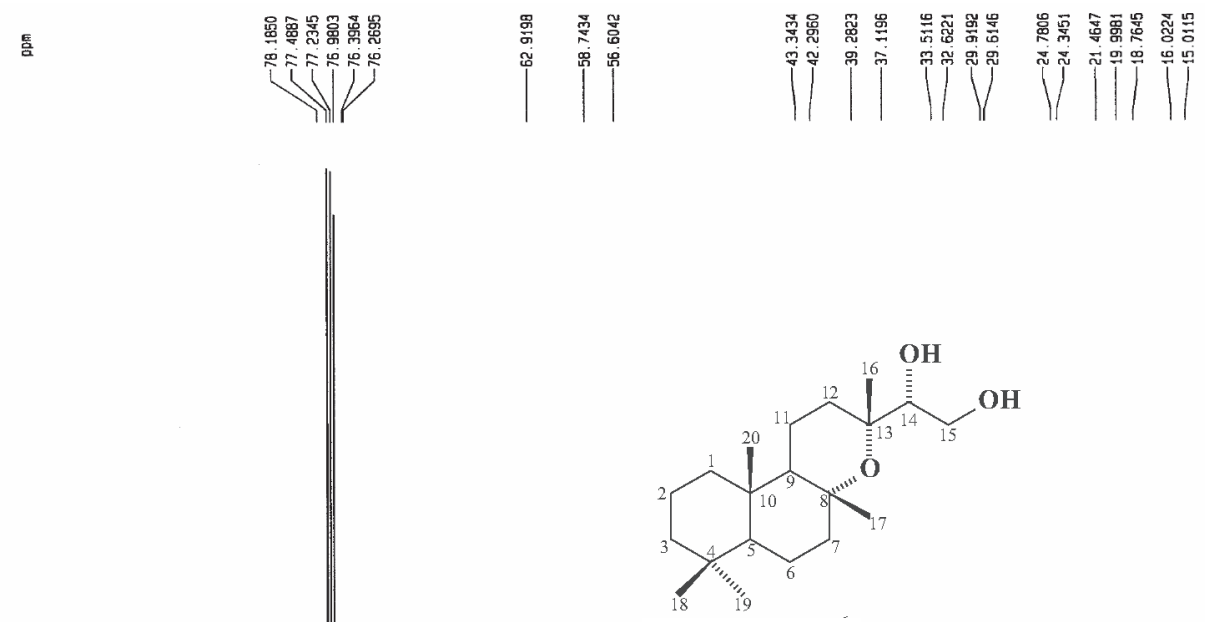

Tarapacol

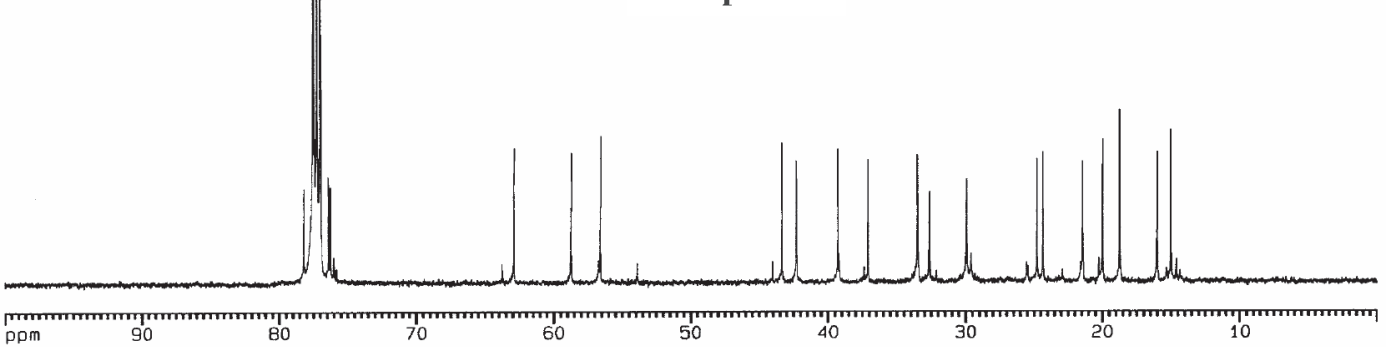

Figure S26. ${ }^{13} \mathrm{C}-\mathrm{BB}$ NMR spectrum $\left(125 \mathrm{MHz}, \mathrm{CDCl}_{3}\right)$ of Tarapacol. 


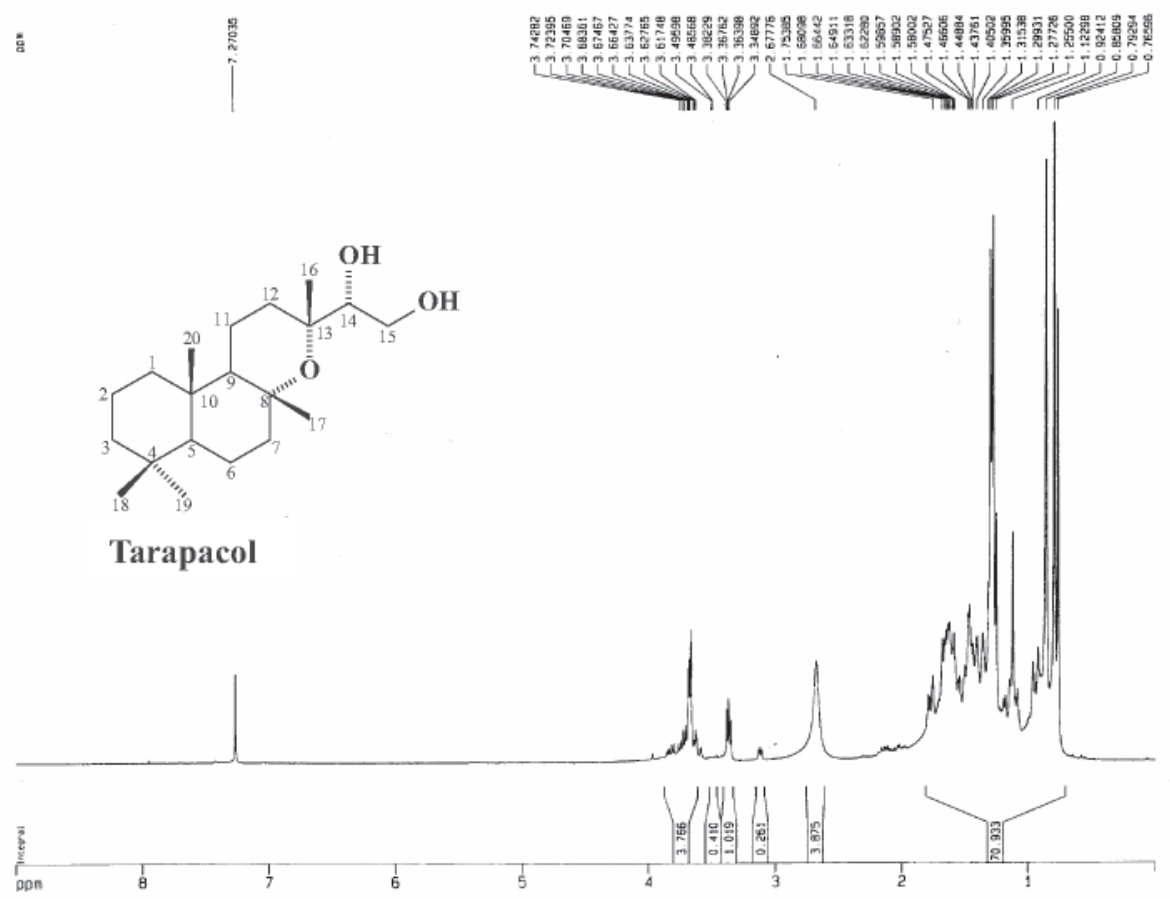

Figure S27. ${ }^{1} \mathrm{H}$ NMR spectrum (500 MHz, $\left.\mathrm{CDCl}_{3}\right)$ of Tarapacol.

言

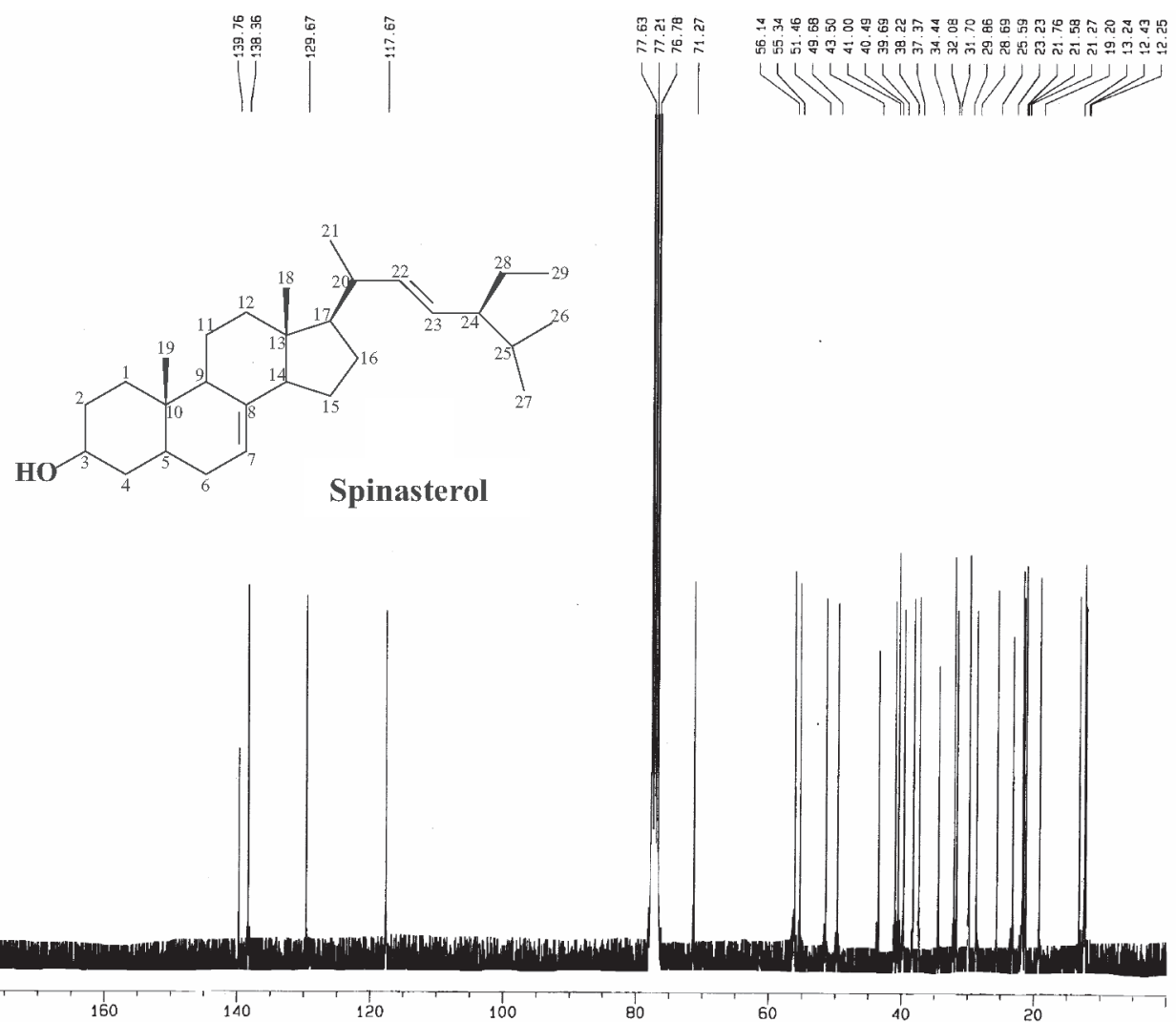

Figure S28. ${ }^{13} \mathrm{C}-\mathrm{BB}$ NMR spectrum $\left(75 \mathrm{MHz}, \mathrm{CDCl}_{3}\right)$ of Spinasterol. 


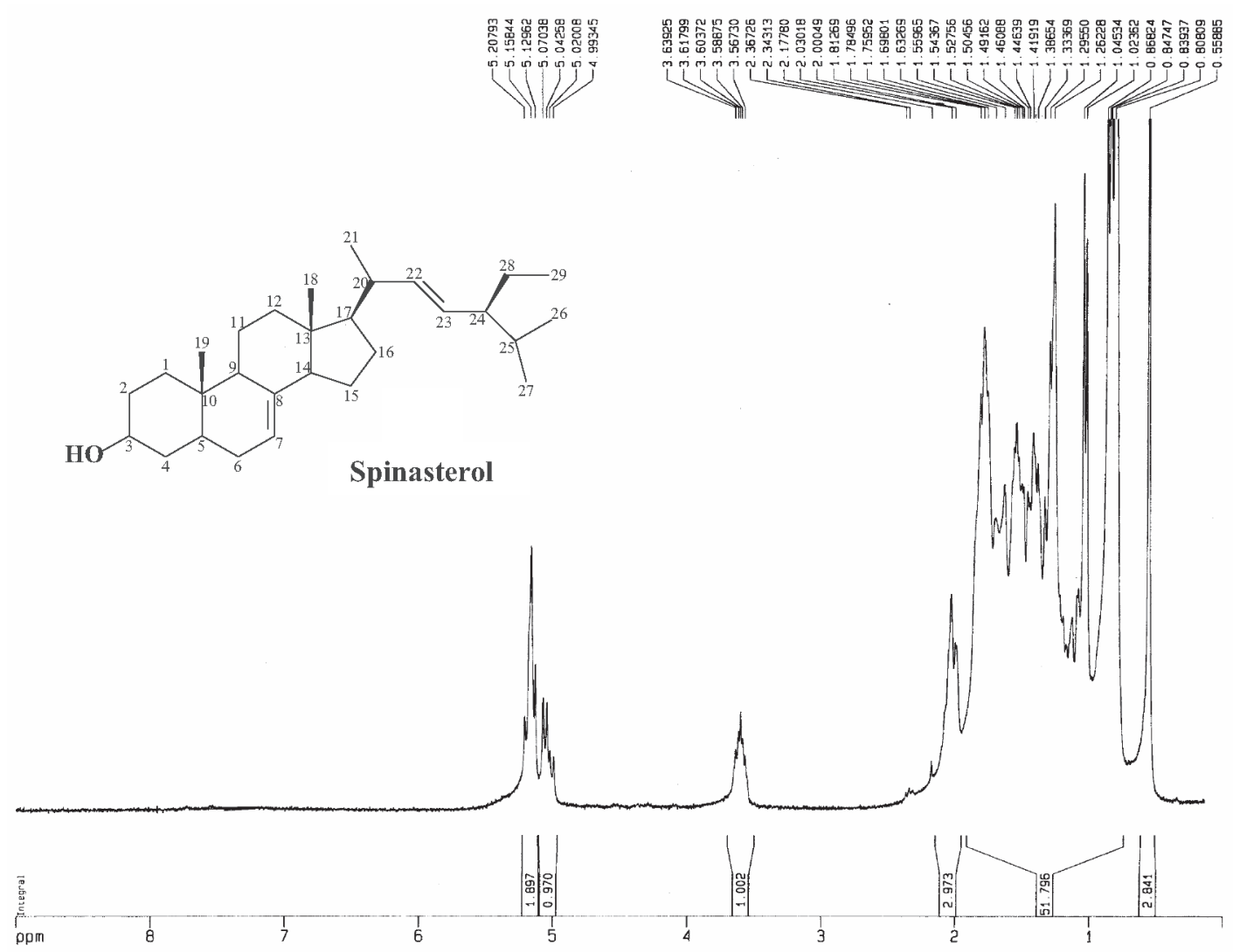

Figure S29. ${ }^{1} \mathrm{H}$ NMR spectrum ( $\left.300 \mathrm{MHz}, \mathrm{CDCl}_{3}\right)$ of Spinasterol.

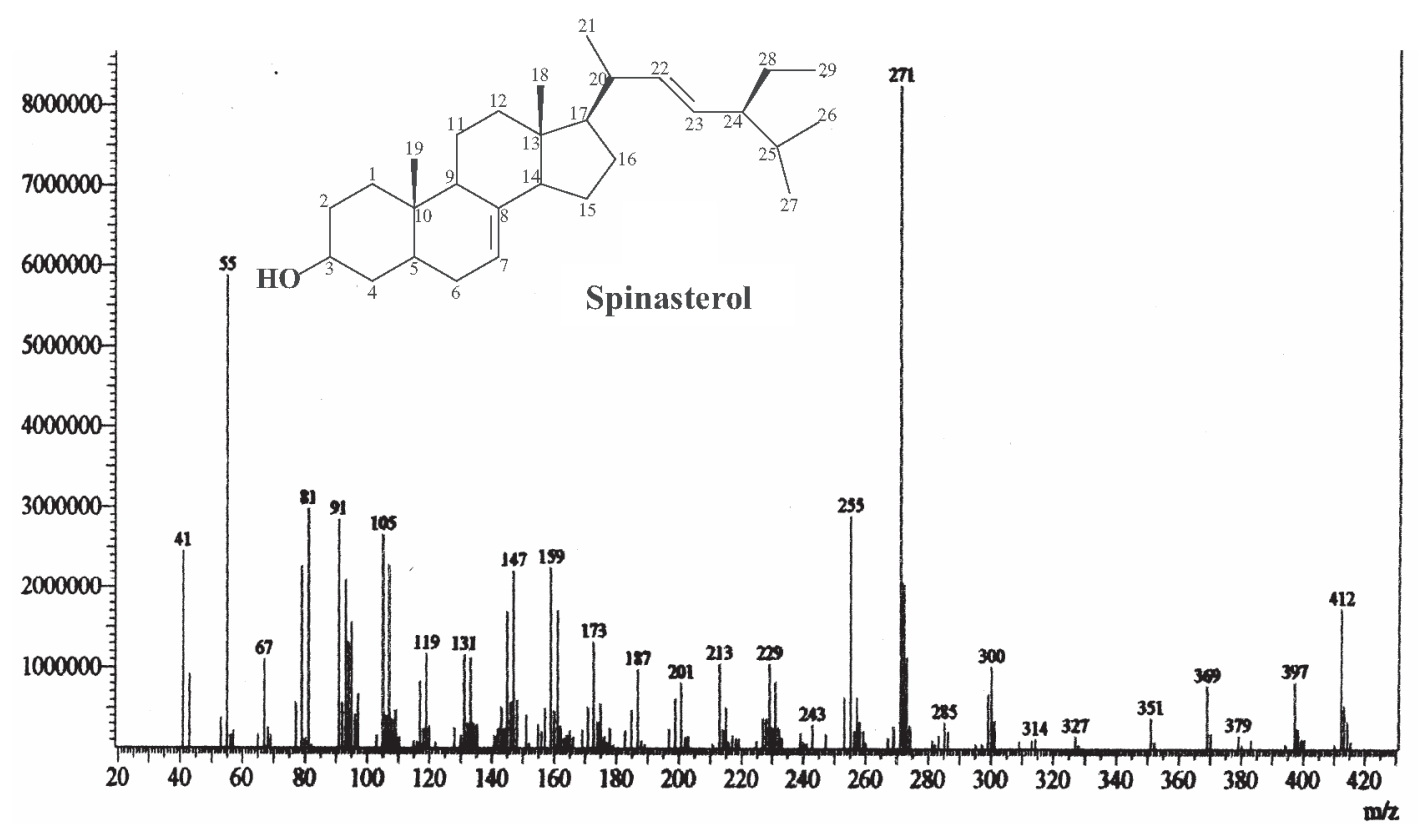

Figure S30. HR-EIMS spectrum of Spinasterol. 


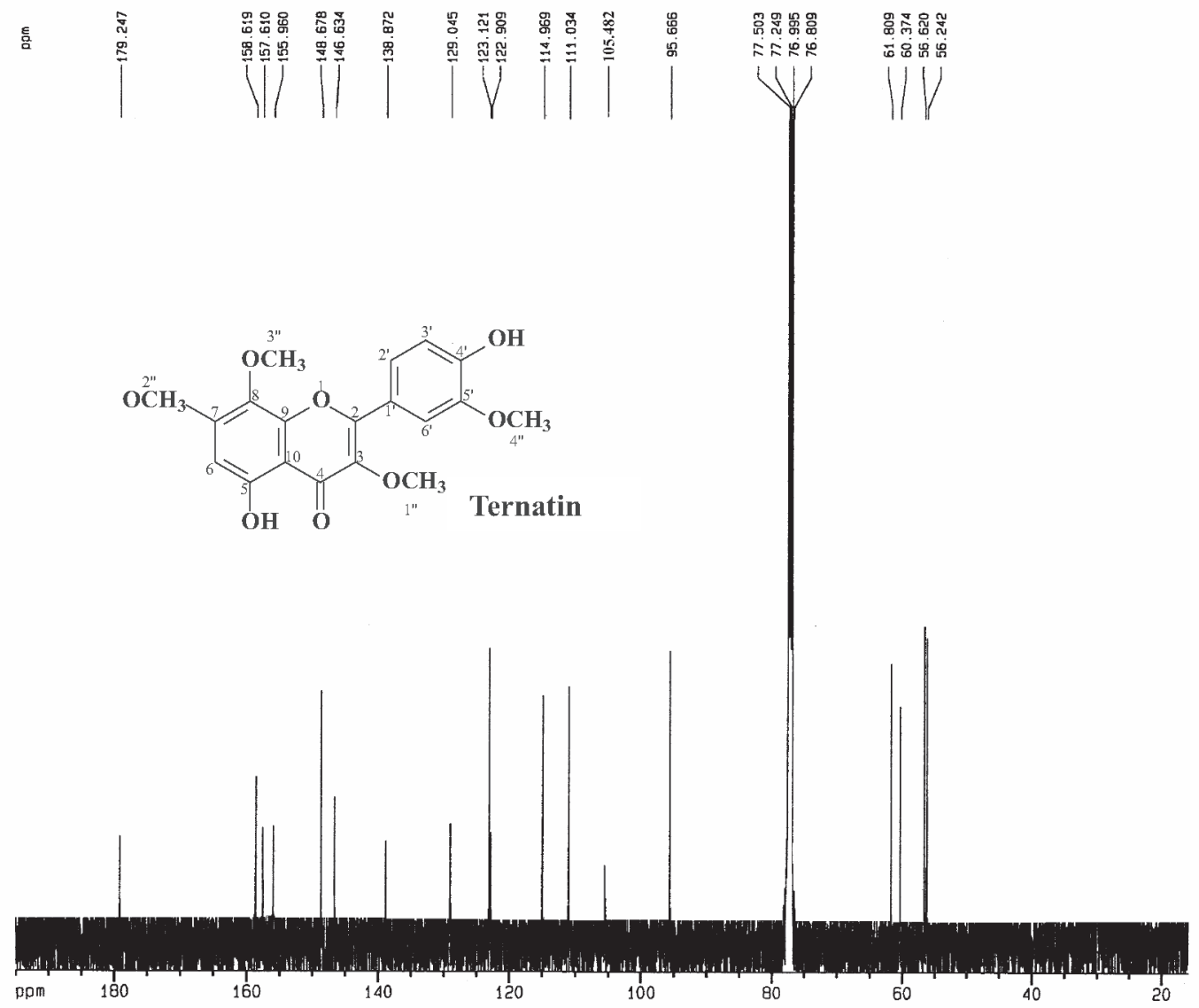

Figure S31. ${ }^{13} \mathrm{C}-\mathrm{BB}$ NMR spectrum $\left(125 \mathrm{MHz}, \mathrm{CDCl}_{3}\right)$ of Ternatin.

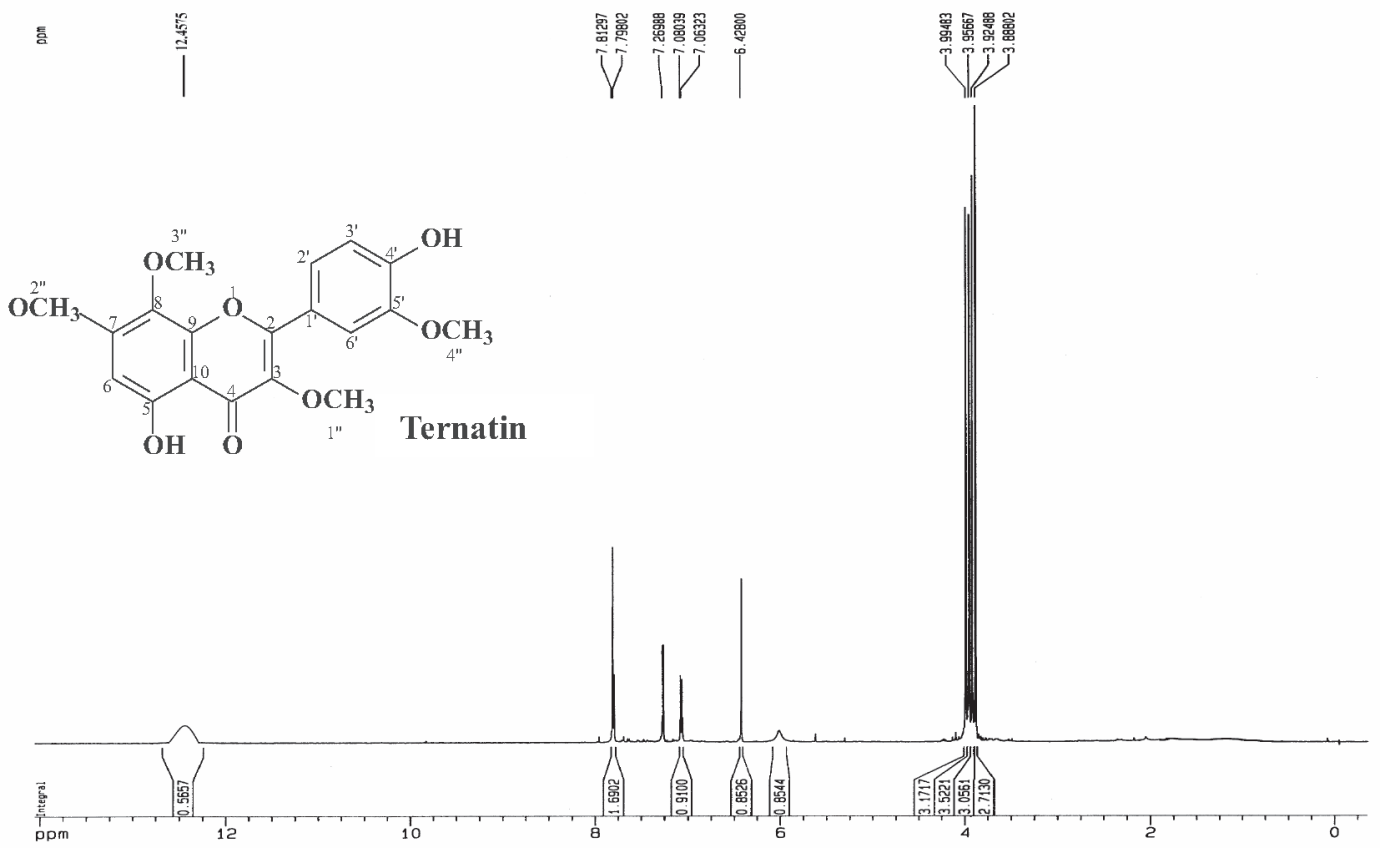

Figure S32. ${ }^{1} \mathrm{H}$ NMR spectrum $\left(500 \mathrm{MHz}, \mathrm{CDCl}_{3}\right)$ of Ternatin. 


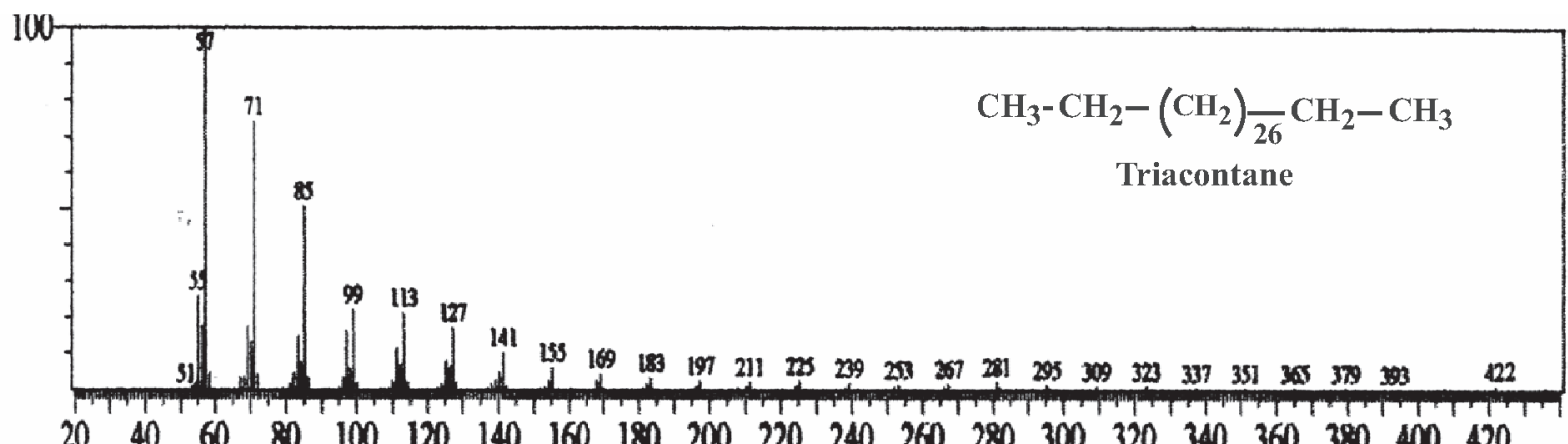

Figure S33. HR-EIMS spectrum of Triacontane.

言

$\mathrm{CH}_{3}-\mathrm{CH}_{2}-\left(\mathrm{CH}_{2}\right)_{26} \mathrm{CH}_{2}-\mathrm{CH}_{3}$

Triacontane

ppom

Figure S34. ${ }^{1} \mathrm{H}$ NMR spectrum $\left(500 \mathrm{MHz}, \mathrm{CDCl}_{3}\right)$ of Triacontane. 


$$
\begin{gathered}
\mathrm{CH}_{3}-\mathrm{CH}_{2}-\left(\mathrm{CH}_{2}\right)_{26} \mathrm{CH}_{2}-\mathrm{CH}_{3} \\
\text { Triacontane }
\end{gathered}
$$

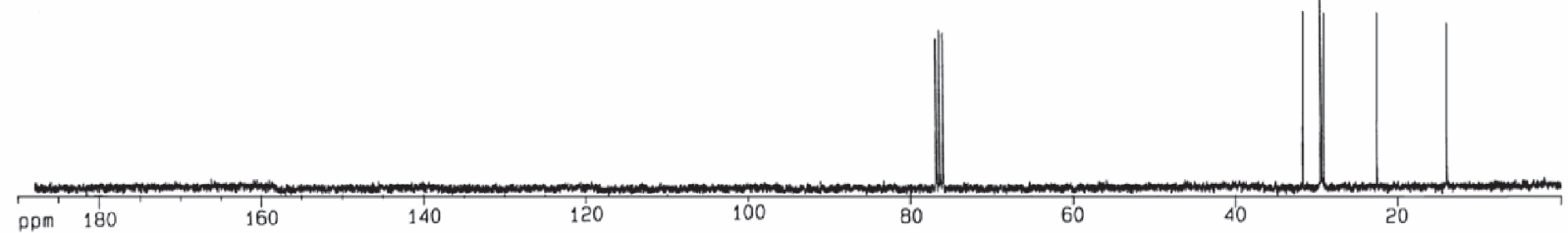

Figure S35. ${ }^{13} \mathrm{C}-\mathrm{BB}$ NMR spectrum $\left(125 \mathrm{MHz}, \mathrm{CDCl}_{3}\right)$ of Triacontane. 